\title{
Familiarity Detection and Memory Consolidation in Cortical Assemblies
}

\author{
Xiaoyu Zhang, ${ }^{1,2}$ Fang-Chin Yeh, ${ }^{1}$ Han Ju, ${ }^{1}$ Yuheng Jiang, ${ }^{1}$ Gabriel Foo Wei Quan, ${ }^{1}$ and \\ (1)Antonius M.J. VanDongen ${ }^{1}$
}

https://doi.org/10.1523/ENEURO.0006-19.2020

${ }^{1}$ Program for Neuroscience and Behavioral Disorders, Duke-NUS Medical School, 169857, Singapore and ${ }^{2}$ NUS Graduate School for Integrative Sciences and Engineering, National University of Singapore, 117456, Singapore

\begin{abstract}
Humans have a large capacity of recognition memory (Dudai, 1997), a fundamental property of higher-order brain functions such as abstraction and generalization (Vogt and Magnussen, 2007). Familiarity is the first step towards recognition memory. We have previously demonstrated using unsupervised neural network simulations that familiarity detection of complex patterns emerges in generic cortical microcircuits with bidirectional synaptic plasticity. It is therefore meaningful to conduct similar experiments on biological neuronal networks to validate these results. Studies of learning and memory in dissociated rodent neuronal cultures remain inconclusive to date. Synchronized network bursts (SNBs) that occur spontaneously and periodically have been speculated to be an intervening factor. By optogenetically stimulating cultured cortical networks with random dot movies (RDMs), we were able to reduce the occurrence of SNBs, after which an ability for familiarity detection emerged: previously seen patterns elicited higher firing rates than novel ones. Differences in firing rate were distributed over the entire network, suggesting that familiarity detection is a system level property. We also studied the change in SNB patterns following familiarity encoding. Support vector machine (SVM) classification results indicate that SNBs may be facilitating memory consolidation of the learned pattern. In addition, using a novel network connectivity probing method, we were able to trace the change in synaptic efficacy induced by familiarity encoding, providing insights on the long-term impact of having SNBs in the cultures.
\end{abstract}

Key words: consolidation; microcircuits; neuronal network; optogenetics; recognition memory

\section{Significance Statement}

Studies of memory mechanisms in neuronal networks formed by cultured neurons have been complicated by spontaneously occurring synchronized network bursts (SNBs), which prevent encoding of stimulus information. We have developed an optogenetic method to suppress SNBs using random dot stimuli, which allowed us to demonstrate the ability of cultured cortical networks to detect a familiar input. Whereas SNBs interfere with familiarity encoding, they may facilitate memory consolidation. These results indicate that generic cortical microcircuits have an innate ability for familiarity detection, a form of recognition memory.

\section{Introduction}

Learning and memory are indispensable for brain functionality. Recognition, the ability to recognize previously experienced sensory inputs, is a form of declarative memory that is common in our daily life and fundamental to many higher-order processes such as concept learning,

\footnotetext{
Received January 7, 2019; accepted February 20, 2020; First published March $2,2020$.

The authors declare no competing financial interests.
}

abstraction and generalization. Prior studies (Nickerson, 1965; Shepard, 1967; Standing, 1973) have demonstrated that subjects can recognize many thousands of pictures several days after they have seen the pictures only once, suggesting a vast recognition memory for images, with no capacity limit detected in practical experiments.

Author contributions: X.Z., F.-C.Y., and A.M.J.V. designed research; X.Z., F.-C.Y., Y.J., and G.F.W.Q. performed research; X.Z., F.-C.Y., H.J., and Y.J. analyzed data; X.Z., F.-C.Y., and A.M.J.V. wrote the paper. 
Familiarity and recollection are two components of recognition memory (Yonelinas, 2002; Squire et al., 2007). Recollection requires accurate recall of details with their context, whereas familiarity merely needs a signal indicating that the object has been encountered before (Wixted, 2007). Unlike conditional or reinforcement learning, which requires association or reward, familiarity is simply encoded through unsupervised learning (i.e., sensory experience) and retrieved on re-presentation of the input. These features have made familiarity a perfect paradigm to study learning and memory from a bottom-up perspective.

Synaptic plasticity has long been implicated in the processes of learning and memory. Memories are thought to be encoded and stored in "engrams," groups of neurons connected through synapses whose efficacy was modified during learning. Characterization of the changes in a population of neurons following learning and after memory formation can be an important building block to forge a link between molecular/cellular synaptic plasticity and behavioral modifications.

Dissociated neuronal cultures growing on multielectrode arrays (MEAs) provide an accessible way to study familiarity in neuronal populations in vitro. MEAs are designed to obtain parallel recordings at multiple sites of the dynamics of cultured neuronal networks. By introducing the light-gated ion channel channelrhodopsin-2 (ChR2) into neurons, non-invasive optogenetic stimulation has been made possible for encoding sensory experiences. Correlation between sensory input and network activity modification can be established to provide insights into the mechanisms of learning and memory at the neuronal network level.

A few groups have already made efforts to study learning and memory using MEAs. Maeda et al. (1998) and Jimbo et al. (1998) found that the reliability and reproducibility of stimuli-evoked network bursts was enhanced after tetanization at single electrodes. Tateno and Jimbo (1999) reported improved temporal precision of initial response spikes to test stimuli after training. Later Jimbo et al. (1999) reported that single-site stimulation could induce pathway-dependent potentiation or depression. However, subsequent attempts made to reproduce these observations were less successful. By including control recordings, Wagenaar et al. (2006b) argued that the "positive modifications" observed did not differ significantly from what could be caused by the drift in spontaneous network activity. Applying the same tetanization protocol ( $20 \mathrm{~Hz}$ delivered at a single site, as in Jimbo et al., 1999), Chiappalone et al. (2008) failed to observe significant potentiation; instead, they saw only a global decrease in the

This work was supported by the Ministry of Education (MOE) Singapore Grant MOE2012-T2-1-039 and the MOH National Medical Research Council Grant OFIRG16May052.

Correspondence should be addressed to Antonius M. J. VanDongen at antonius.vandongen@duke-nus.edu.sg.

https://doi.org/10.1523/ENEURO.0006-19.2020

Copyright (C) 2020 Zhang et al.

This is an open-access article distributed under the terms of the Creative Commons Attribution 4.0 International license, which permits unrestricted use, distribution and reproduction in any medium provided that the original work is properly attributed. evoked network activity (Chiappalone et al., 2008, their Fig. 4). Additional studies were conducted, either with electrical (Massobrio et al., 2015) or optogenetic stimulation (Lignani et al., 2013). Constrained by the inflexibility of input and output (electrical stimulation and data collection through the same electrodes), most of the studies characterized network dynamics by measuring changes in mean firing rate, as well as burst frequency and duration, the values of which are highly variable and poorly associated with learning. Consequently, results from these studies have not reached a common agreement to date. Whether in vitro neuronal networks exhibit learning and memory as an emerging property remains inconclusive.

Computer simulations with neural network models previously demonstrated that generic cortical microcircuits with bidirectional synaptic plasticity can perform familiarity detection (Zhang et al., 2017). However, these simulated networks do not have all the properties of neuronal networks growing on MEAs. One major difference is the synchronized network bursts (SNBs), which are universally observed in biological cultures. A SNB is characterized by networkwide synchronized high-frequency firing that is spontaneously initiated and lasts several hundred milliseconds. SNBs occur periodically in cultured cortical networks in the absence of external inputs, and are separated by windows of nearly silent network activity with occasional sparse asynchronous firings (Maeda et al., 1995; van Pelt et al., 2004; Eytan and Marom, 2006; Chiappalone et al., 2007; Colombi et al., 2016). In vitro studies investigating the capability of neuronal networks to process spatiotemporal inputs have shown that SNBs disrupt short-term memory (Dranias et al., 2015; Ju et al., 2015). Similarly, when SNBs were introduced in simulated neural networks, average performance on familiarity detection declined (Zhang et al., 2017). In this study, we provide evidence on familiarity detection in MEAs in the presence of SNBs.

\section{Materials and Methods}

\section{Culture preparation}

Dissociated cortical primary cultures were prepared from Sprague Dawley rat embryonic day (E)18 brains from either sex. Cortical tissue was dissected in ice-cold HBSS. The isolated cortices were digested using the Worthington Papain Dissociation System (Worthington Biochemical Corporation). Cells were plated on MEA dishes with 252 electrodes arranged in a $16 \times 16$ grid $(30-\mu \mathrm{m}$ diameter, $200-\mu \mathrm{m}$ interelectrode distance, Multi-Channel Systems). Prior to plating, MEAs were cleaned with 1\% Tergazyme solution, sterilized with $70 \%$ ethanol, surface-treated with fetal bovine serum (FBS), and coated with $0.1 \mathrm{mg} / \mathrm{ml}$ polyD-lysine (Invitrogen). Cells were plated onto a circular area with a diameter of $6 \mathrm{~mm}$ centered at the electrode array area. The final density was $\sim 4000$ cells $/ \mathrm{mm}^{2}$. The plating droplet was left on the MEAs for $30 \mathrm{~min}$ to allow cell attachment. Culturing medium (Neurobasal supplemented with 2\% B-27, 0.5 mm L-glutamine, 10\% penicillin/streptomycin) with $10 \%$ FBS (Sigma) was then added to the cells (1 $\mathrm{ml}$ for each MEA). Medium was completely changed $24 \mathrm{~h}$ later [1d in vitro (DIV1)] to remove FBS. Subsequent 
medium changes were done on DIV6, DIV10, and every $3 \mathrm{~d}$ afterwards. Half of the medium was replaced each time. Cultures were covered with plastic caps with a fluorinated ethylene-propylene membrane (ALA-Scientific) and maintained in a humidified $\mathrm{CO}_{2}$ incubator $\left(5 \% \mathrm{CO}_{2}, 37^{\circ} \mathrm{C}\right)$. On DIV9, MEA cultures were transfected with an adeno-associated virus (AAV9) encoding ChR2 (AAV9.hSyn.hChR2 (H134R)-EYFP.WPRE.hGH, Addgene 26973P, MOI 100,000). Half of the medium was changed on DIV10 to prevent virus toxicity. Alternative transfection on DIV1 was applied on cultures used in the study of early connectivity development. Overall, we observed similar development patterns between cultures transfected on DIV1 and DIV9. DIV9 was chosen as the transfection date for cultures used for the main experiments for its least disruption on long-term viability of the cultures. All experimental procedures were conducted in accordance with Institutional Animal Care and Use Committee (IACUC) and approved by SingHealth.

\section{MEA recording}

Recordings were performed on an anti-vibration table and in a Faraday cage. During recordings, MEAs with culturing medium (see above, Culture preparation) were placed in a customized $\mathrm{CO}_{2}$ incubator placed on top of an inverted microscope (see below, Optical stimulation) inside the cage. Extracellular electrophysiological signals were acquired using the USB-MEA256 hardware systems (Multi Channel Systems). MC_Rack software (Multichannel Systems) was used to process extracellular signals that were high pass filtered at $300 \mathrm{~Hz}$ and low pass filtered at $3 \mathrm{kHz}$ with fourth-order Bessel filters. Each channel was sampled at a frequency of $20 \mathrm{kHz}$. Action potentials were detected using a voltage threshold set at six times the SD $(6 \sigma)$ of the biological noise for each recording channel.

\section{Optical stimulation}

Stimulus presentations and MEA recordings were triggered and synchronized by transistor-transistor logic (TTL) pulses generated by MATLAB that signaled the beginning and end of each session. Optical stimulation was conducted using a 500-mW DPSS laser with a wavelength of $473 \mathrm{~nm}$. The laser beam was optically expanded and projected onto a reflective spatial light modulator (SLM; Holoeye Photonics), with a resolution of $1920 \times$ $10^{80}$ pixels. The patterns reflected by the SLM were controlled by the DVI graphics output of a personal computer and were refreshed at $50 \mathrm{~Hz}$. The reflected light patterns were then projected onto MEA cultures through the objective lens of an inverted microscope (Eclipse Ti-E, Nikon). The final light intensity at the MEA culture is $\sim 4.5 \mathrm{~mW} /$ $\mathrm{mm}^{2}$. This setup allowed us to design arbitrary blue-light images and use them as stimuli. The stimulation presentation was programmed with the Psychtoolbox-3 (http:// psychtoolbox.org) in MATLAB.

\section{Cross-correlation histogram $(\mathrm{CCH})$ probing}

Cultured neuronal networks were probed with optical stimuli to identify synaptic connections and monitor changes in their efficacies. The optical stimulation area, which approximately covered the electrode array area, is divided into a $16 \times 16$ grid, resulting in 256 square "dots." Each probing session is conducted by stimulating the culture with a series of random-dot frames, in which all grid positions were black, except for five randomly-selected positions which were white, resulting in five blue dots simultaneously stimulating the network when the laser is on. Each stimulating frame lasts $100 \mathrm{~ms}$, during which the light is on for $40 \mathrm{~ms}$, and off for $60 \mathrm{~ms}$. Each of the 256 positions in the grid is stimulated 20 times in total in a random order during a probing session, resulting in a random dot movie (RDM) of 5120 frames. Each probing session lasts $\sim 1.7 \mathrm{~min}$. Neuronal responses were recorded from the 252 MEA electrodes concurrently with the optogenetic random dot probing. A potential causal relationship between each stimulating grid position and each electrode (recording position) was evaluated by calculating a $\mathrm{CCH}$ between the electrode response time-series and the stimulating time-series. CCHs are calculated as

$$
\operatorname{cch}(\tau)=\sum_{t} i(t) j(t-\tau)
$$

where $\tau$ is the time lag between the two time-series, and $i$, jare the time-series representations (1-dimensional vectors) of the electrode response time points and the stimulus time points. The entire probing session was binned into 1-ms windows. For an electrode, if it detects spikes in a particular time bin, the time-series vector $i$ will contain 1 in the corresponding time bin, otherwise 0 . For a stimulating position, if a probing stimulus is imposed in a particular time bin, the time-series vector $j$ will contain 1 in the corresponding time bin, otherwise 0 . $\mathrm{CCH}$ calculates the discrete cross-correlation between the two time-series and find the time lag that returns the largest correlation (the peak of the $\mathrm{CCH}$ curve). A sharp peak with a positive time lag in the $\mathrm{CCH}$ indicates that the electrode consistently detects a response at a defined time window after the grid position is stimulated, and therefore a directional synaptic connection likely exists between the grid position (presynaptic) and the electrode (postsynaptic). Assuming the recorded spike train (electrode response) follows a Poisson process, the significance of the peak can be tested against the $p$ value of the Poisson distribution:

$$
P(k)=e^{-\lambda} \frac{\lambda^{k}}{k !} .
$$

$P(k)$ computes the chance of observing a peak value of $k$ given the mean of $\operatorname{cch}(\tau)$ as $\lambda$. When the chance falls below $\mathrm{P}=1 \mathrm{e}^{-6}$, and the electrode detects a response in more than half of the trials (10 out of 20 times when the dot is stimulated), we draw a connection. The value $1 \mathrm{e}^{-6}$ is chosen empirically to control the number of connections that will be detected as reliable connections in a culture. Setting it too high $\left(1 \mathrm{e}^{-5}\right)$ will result in having too many connections detected, e.g., some channels are constantly firing and thus will be assigned connections with nearly all stimulating position. Setting it too low $\left(1 \mathrm{e}^{-7}\right)$ will result in having too few connections detected and 
increasing the variance of network analysis. The latency of the connection is reflected by the time lag $\tau_{p}$ where the $\mathrm{CCH}$ reaches its peak after stimulation. The efficacy of the connection is estimated by the area under the poststimulus time histogram (PSTH). With the detected connections, we were able to draw a connectivity map for each culture. The connectivity map comes together with directionality information, which can be used to augment analysis and understanding. Two connectivity maps of the same culture obtained before and after a certain treatment can be compared with evaluate whether the culture has undergone significant connectivity changes. For instance, the relative change of the efficacy, change in the area under the PSTH curves divided by area under the initial PSTH curve, is calculated for all connections. Positive efficacy change indicates potentiation, while negative efficacy change indicates depression. To evaluate the overall network efficacy change, the positive changes and negative changes are summed together respectively over all connections and represented as "P: $\sum(0<$ changes $<100 \%+$ changes $>100 \%$ )" and "N: $\sum$ (changes $<0 \%$ )" in the figure legend. The unit of $\mathrm{P}$ and $\mathrm{N}$ is [\%]. Positive changes $>100 \%$ are capped at $101 \%$. The potentiation to depression ratio " $R$ " $(R=P /|N|$, where $|N|$ is the absolute value of $\mathrm{N}$ ) is calculated to reflect the overall change in network efficacy. $\mathrm{R}>1$ indicates long-term potentiation (LTP) at the network level and $\mathrm{R}<1$ indicates long-term depression (LTD) at the network level. $\mathrm{CCH}$ probings were conducted 5 min after each treatment to reflect the longterm effect associated with the treatments.

\section{Experimental design}

We used three cartoon-like images of a car front, a dog face and a human face (Fig. $1 H$ ) to study the networks' ability to learn and memorize complex input patterns. The three patterns were converted to line drawings in $50 \times 50$ grids of the same size as the stimulating area. The luminance of each pattern is tuned to balance the elicited neuronal baseline response. Experiments typically consisted of three sessions: baseline recording, learning stimulation and testing recording. Network baseline responses to each of the three patterns was recorded 10 times. Three patterns were presented in random orders with 10-s intervals and 100-ms stimulation time. As the network response is subject to noise introduced by fluctuation in the network background activity level due to SNBs, applying an illuminating window of $100 \mathrm{~ms}$ enables us to record stable and consistent network responses for baseline and testing phases. Learning was conducted by stimulating the cultures with one of the three patterns at $50 \mathrm{~Hz}(50 \%$ duty cycle, $10 \mathrm{~ms}$ on and $10 \mathrm{~ms}$ off) for 60 trials with 9-s intervals and 1-s stimulation time. Network testing (postlearning) response was recorded the same way as baseline recording.

\section{Burstiness index (BI)}

We adopted the idea of $\mathrm{BI}$ (Chiappalone et al., 2005; Hinard et al., 2012) to quantify degree of network bursting. Continuous recordings of network spontaneous activity
(10 min, recorded at the beginning of experiments) were divided into 1-s bins and the fraction of spikes contained by the $15 \%$ most active bins was calculated (f15). BI was defined as $\mathrm{BI}=(\mathrm{f} 15-0.15) / 0.85$, with 0 for not bursting at all, and 1 for maximum burstiness. The $15 \%$ was chosen empirically with the observation that SNBs occur on average in $15 \%$ of the time bins in an SNB-dominant culture. Equivalently it assumes the culture has an average interburst interval (IBI) of $6.7 \mathrm{~s}$. For cultures with average $\mathrm{IBI}<6.7 \mathrm{~s}$, we increased the $15 \%$ accordingly.

\section{RDMs}

In order to suppress SNBs, neuronal networks were pre-stimulated by RDMs. In experiments involving RDM treatment, the movie was generated in a $10 \times 10$ grid of the same size as the stimulating area. In each frame, 25 out of 100 dots were randomly chosen and illuminated to stimulate the culture for $50 \mathrm{~ms}$. The culture was then allowed to rest for $50 \mathrm{~ms}$ in dark. Frames were refreshed at a frequency of $10 \mathrm{~Hz}$. These values for the frequency and number of dots were determined to be optimal for suppression of SNBs. RDMs were played for $0.5-1 \mathrm{~h}$ to the cultures, after baseline recording, and before learning stimulation.

\section{Culture exclusion}

For experiments comparing the conventional learning paradigm and the RDM-learning paradigm (Figs. 7G,H, 8), we started with 26 healthily plated cultures (enough cells survived by DIV22 for good coverage of the stimulating area). Before each experiment, we recorded a 10-min session of the culture's activity at rest and excluded cultures with frequency of background SNBs $>0.2 \mathrm{~Hz}$ (too active) or $<0.05 \mathrm{~Hz}$ (too quiet). Five cultures were excluded this way. Cultures whose network efficacy changed significantly before and after the baseline/testing recording phase were also excluded, because they were found to be unstable and produce inconsistent results in other steps. Five cultures were excluded this way. For the RDM learning paradigm, cultures whose bursting activity was not significantly suppressed by the RDMs $(R>0.2)$ were excluded from the study, because their subsequent learning capacity were compromised. Three cultures were excluded this way. Eventually we were left with a total of thirteen cultures: the conventional learning paradigm was used for five cultures, in three cultures we used the RDMlearning paradigm and conducted testing $10 \mathrm{~min}$ after the learning stimulation, while in five cultures we used the RDM-learning paradigm with extended testing at $30 \mathrm{~min}$, $1 \mathrm{~h}$, and $24 \mathrm{~h}$ after learning.

\section{Support vector machine (SVM) classification}

Pattern-induced network responses at baseline and testing phases are binned at $10 \mathrm{~ms}$. Data from all bins of the three classes (human, dog, car) are pooled together (pooling-all-sample method) to train one SVM classifier, to avoid the curse of dimensionality (the number of features is greater than the number of instances). The trained SVM classifier was then applied to classify 5-min 

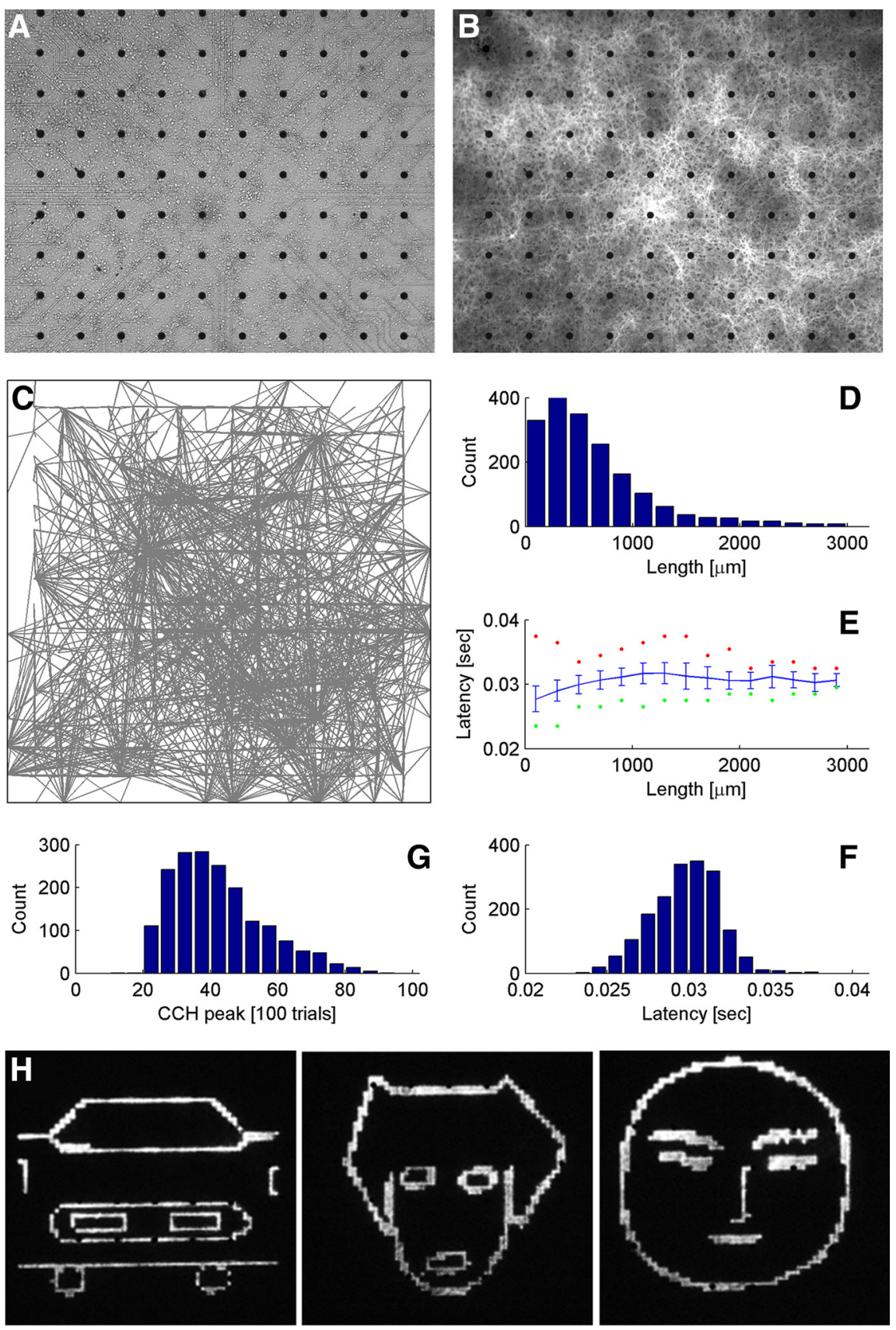

Figure 1. Network connectivity and stimulus pattern. $\boldsymbol{A}, \boldsymbol{B}$, Pictures of a DIV30 MEA culture taken with a $4 \times$ objective illuminated by bright-field light $(\boldsymbol{A})$ and fluorescent light $(510 \mathrm{~nm}$ with 3 -s exposure time) to visualize ChR2-YFP expression (B). $\boldsymbol{C}-\mathbf{G}$, Network connectivity summary of the MEA culture. $\mathbf{C}$, All significant connections detected by $\mathrm{CCH}$ probing. Each line is drawn from a presynaptic dot position to a postsynaptic electrode. $\boldsymbol{D}$, Histogram of the distances of the connections in $\boldsymbol{C}$, binned at $200 \mu \mathrm{m}$, which is the spacing between two adjacent electrodes. $\boldsymbol{E}$, Group average latency of the corresponding connections in $\boldsymbol{D}$. Error bars indicate the SDs. Max and min latencies are marked as red and green. $\boldsymbol{F}$, Histogram of the latencies of the connections. $\boldsymbol{G}$, Histogram of the $\mathrm{CCH}$ peak values of the connections. The value is normalized to 100 trials. A peak at 100 means for every trial when the presynaptic dot position was stimulated, the postsynaptic electrode detected a response. Connection efficacy is positively correlated with the $\mathrm{CCH}$ peak, but to be more precise, we used the area under the PSTH curve as the measure of connection efficacy in the subsequent analyses. $\boldsymbol{H}$, Cartoon-like images of a car front, a dog face and a human face, projected as blue light patterns onto an MEA culture with ChR2-YFP expressing neurons.

continuous recordings of network spontaneous activity (binned at $10 \mathrm{~ms}$ ) obtained before the baseline phase and immediately after the learning phase. Classified labels during SNBs were summarized to get the ratio of classified SNB bins belonging to each of the three complex patterns. In a separate effort to validate the usage of the pooling-all-sample method, $60 \%$ of the pattern-induced network responses were used to train the SVM 
classifier and the remaining data were classified by the trained classifier. LIBSVM (Chang and Lin, 2011, software available at http://www.csie.ntu.edu.tw/ cjlin/libsvm, RRID:SCR_010243) and its built-in multiclass classification were used.

\section{Evaluation metric}

The learning protocol $(50 \mathrm{~Hz}, 50 \%$ duty cycle, $10 \mathrm{~ms}$ on, and $10 \mathrm{~ms}$ off for 60 trials with 9-s intervals and 1-s stimulation time) was applied equally to all cultures, because we did not set up any feedback loop that can be used to control the learning duration or stop the tetanus stimulation after the induced LTP exceeds a pre-set threshold. As a result, the learning-induced LTP might appear inadequate for some cultures, i.e., they may display similar magnitudes of change induced by "learning" as compared with response fluctuation due to noise. In light of this, the focus of the performance analysis is not the significance level of magnitude of change, but whether the learned pattern triggers more response than the control patterns at testing. In detail, the induced response differences between the familiar pattern and the two control patterns were averaged and then the value was normalized against the response to the familiar pattern to make the result comparable among cultures:

$$
\Delta=\frac{\left(x_{f}-x_{c 1}\right)+\left(x_{f}-x_{c 2}\right)}{2 x_{f}},
$$

where $x$ is the network response to be evaluated. It can take the value of network firing rate induced by the input patterns or the ratio of classified SNB bins belonging to the input patterns [for more information, see above, Support vector machine (SVM) classification]. $x_{f}$ is the response induced by the familiar pattern. $x_{c 1}, x_{c 2}$ are the responses induced by the two control patterns.

\section{Results}

The goal of the experiments described here was to investigate the ability of cultured neuronal networks resembling cortical microcircuits to recognize a familiar (previously seen) stimulus pattern. We have used cultured cortical neurons growing on a MEA and transfected with ChR2, allowing us to optogenetically stimulating the network with complex patterns, while recording from hundreds of neurons (Fig. 1). A universal property of dense cultured cortical networks is the appearance of highly SNBs every few seconds (Maeda et al., 1995; van Pelt et al., 2004; Eytan and Marom, 2006; Chiappalone et al., 2007; Colombi et al., 2016). We have therefore characterized the development of SNB activity in our MEA cultures and developed an optogenetic approach to suppress them. In additional, we developed an optogenetic approach to define directional connectivity maps for the networks, using a $\mathrm{CCH}$ analysis, which allows us to measure alterations in synaptic efficacy following learning paradigms.

\section{Bursting, connectivity, and network development}

Similar to previous observations, SNBs emerged in the MEA cultures on DIV8 and became dominant from DIV13 onwards. The frequency and duration of SNBs increased over time and stabilized after DIV20 (Wagenaar et al., 2006a), with characteristic IBIs of 5-10 s. To trace the neuronal network development, we established longitudinal connectivity maps for the cultures (Fig. $2 A-D$ ). These maps reveal that local connections (length $\leq 300 \mu \mathrm{m}$ ) are detectable at DIV13 (Fig. 2A); a sparsely connected network starts to form by DIV19 (Fig. 2B); global connections (length $>300 \mu \mathrm{m}$ ) become widespread by DIV22 (Fig. 2C); finally, a densely connected network with extensive global and local connections is formed by DIV26 (Fig. 2D). If loosening the threshold used for $\mathrm{CCH}$ detection from $1 \mathrm{e}^{-6}$ to $1 \mathrm{e}^{-5}$ (see Materials and Methods for more details), long-range connections will appear in the connectivity maps at DIV13 (data not shown) and DIV19 (Fig. 2E).

The developmental profile described by Figure $2 A-D$ varied 1 or $2 \mathrm{~d}$ for individual cultures with different cell densities. We performed most of our experiments from DIV22 onwards, so we could characterize learning-induced changes of a large number of connections and understand the effect of a training paradigm from the network perspective. This time window was also consistently used in (Chiappalone et al., 2008). A statistical summary of the number of connections and connection length of Figure $2 A-D$ is provided in Figure $2 F$.

\section{Network response to high-frequency stimuli}

Another benefit of using mature and strongly connected cultures is that they are able to fire more synchronously in response to high-frequency stimuli. The ChR2 version we used has the H134R mutation, which increases the photocurrent but is associated with slower channel-closure kinetics, resulting in a reduced temporal precision (Yizhar et al., 2011). It is shown in the $\mathrm{CCH}$ probing data that the latency of an optically elicited response is centered around $30 \mathrm{~ms}$ (Fig. 1F), which limits the firing frequency to be elicited through direct optical response to $30 \mathrm{~Hz}$. Nevertheless, a subset of neurons in mature cultures was able to fire synchronously to stimuli with a frequency of $>30 \mathrm{~Hz}$ (for examples, see Fig. 2G3-G4). These neurons are usually receiving EPSPs from multiple concurrently stimulated grid positions. We name them as the "postsynaptic hub neurons" in connectivity maps. Strong connection efficacies in mature cultures assist the propagation of EPSPs, thereby securing the temporal precision of firing. Nevertheless, pyramidal neurons have been reported not to follow well with stimuli beyond $40 \mathrm{~Hz}$, because they are limited by their intrinsic cellular biophysical properties (Hjelmstad et al., 1997; Gunaydin et al., 2010). The reliability of neuronal activation through ChR2 dropped significantly as the frequency of light pulse delivery increased (Arenkiel et al., 2007). We tested MEA culture responses to stimuli of $20-100 \mathrm{~Hz}$ (Fig. 2G1G5). The highest frequency that cortical neurons could fire synchronously was $50 \mathrm{~Hz}$. Therefore, we used $50 \mathrm{~Hz}$ as the learning stimulation protocol for subsequent experiments.

\section{Plasticity induction in presence of SNBs}

By looking at the BI values, the cultures can be classified into two types. Cultures dominated by SNBs are 

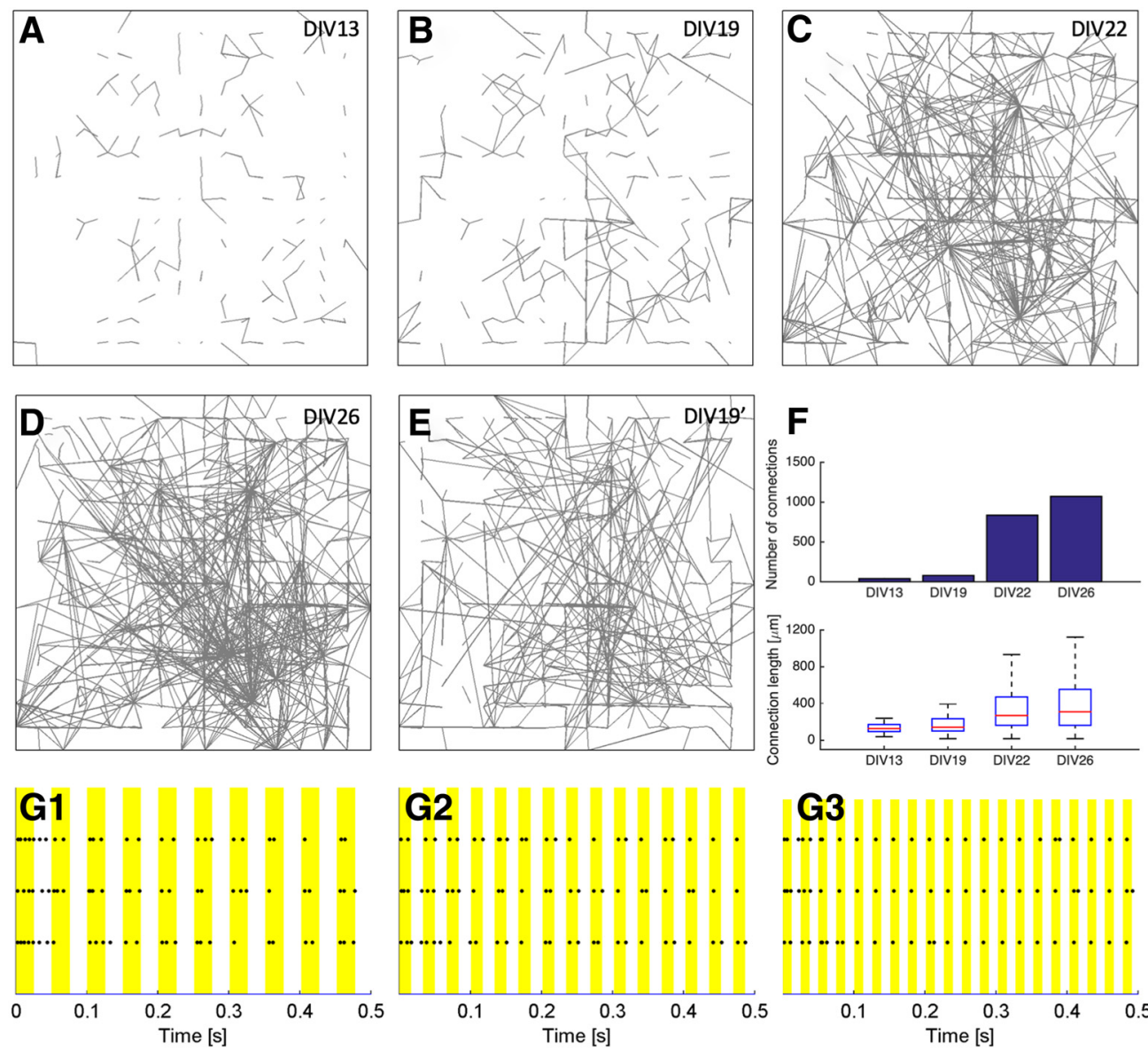

G2
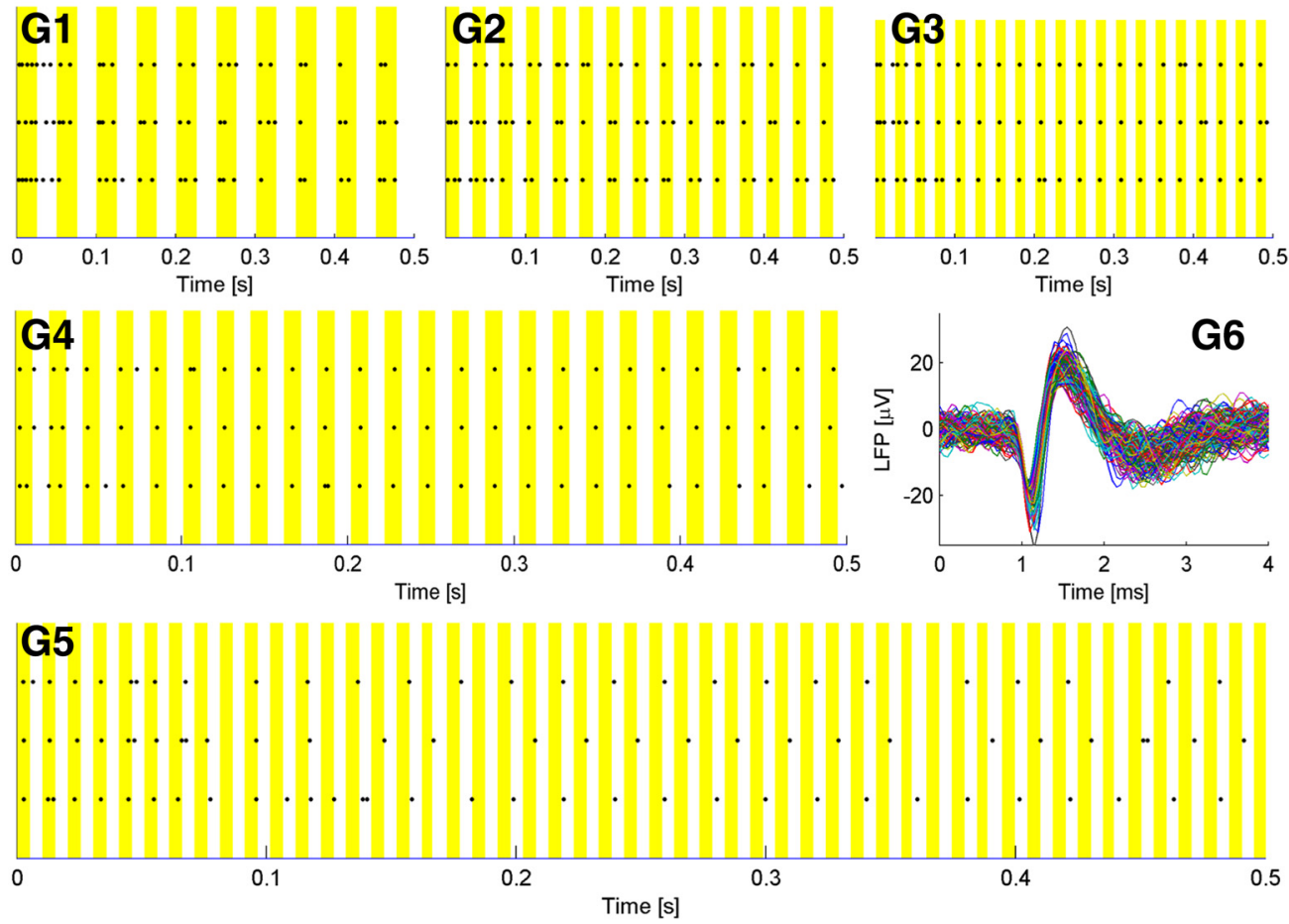

Figure 2. Development of network connections and neuronal responses to high-frequency stimuli. $\boldsymbol{A}-\boldsymbol{D}$, Culture connectivity detected by $\mathrm{CCH}$ probing. The culture was transfected with ChR2-YFP on DIV1. CCH measurements were conducted on DIV13 (A), DIV19 $(\boldsymbol{B})$, DIV22 $(\boldsymbol{C})$, and DIV26 $(\boldsymbol{D})$. $\boldsymbol{E}$, Culture connectivity if loosening the threshold of CCH detection on DIV19. $\boldsymbol{F}$, Summarizing the number of connections detected on DIV13, DIV19, DIV22, and DIV26 (top), and the distribution of connection length for each DIV (bottom). G1-G6, Channel responses to light pulses of $20 \mathrm{~Hz}(\mathbf{G 1}), 30 \mathrm{~Hz}$ (G2), $40 \mathrm{~Hz}($ G3), $50 \mathrm{~Hz}$ (G4), and 100 Hz (G5). G6, Channel waveforms in response to $50-\mathrm{Hz}$ pulses (G4). The channel is selected as a representative of channels with the best response to high-frequency tetanus. Culture was stimulated on DIV22. Yellow bars highlight when light pulses were delivered.

found to be associated with high $\mathrm{BI}$ values $[\mathrm{BI}=0.86 \pm$ 0.03 (SEM), $n=6]$. Cultures with compromised SNBs and more asynchronous firings are associated with low $\mathrm{BI}$ values $[\mathrm{BI}=0.38 \pm 0.08$ (SEM), $n=3]$. After delivering $50 \mathrm{~Hz}$ stimuli to the cultures, we noticed opposite outcomes from the two types of cultures (Fig. 3). In cultures dominated by SNBs, networks exhibited LTD in overall change $[R=0.67 \pm 0.16$ (SEM), $n=6$; for an example, see Fig. 

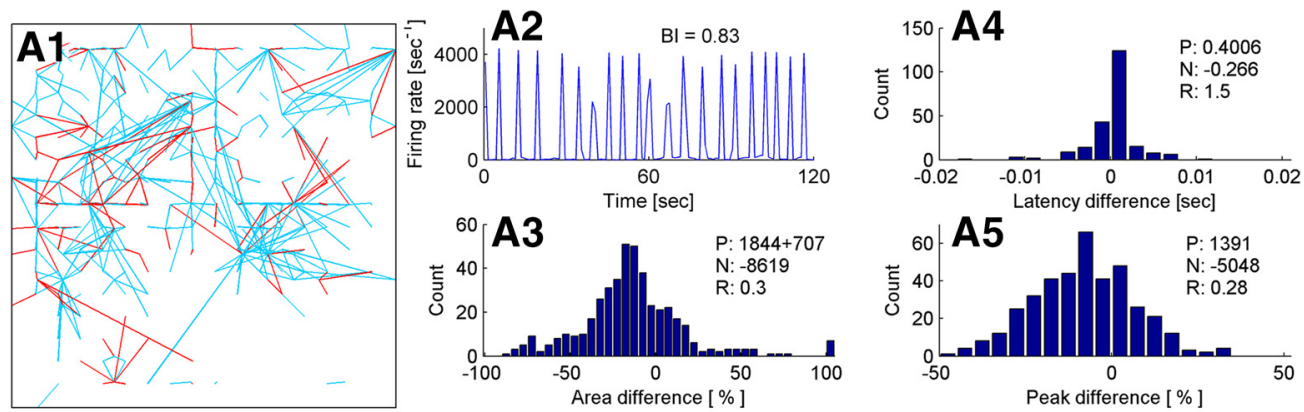

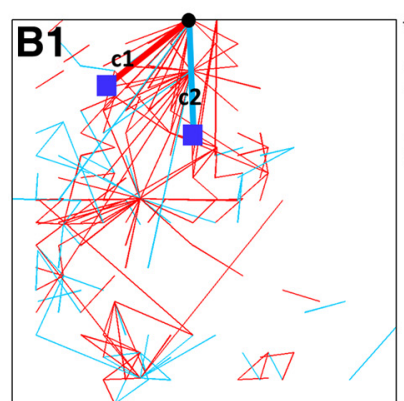

C1
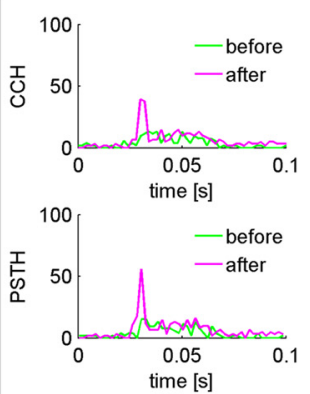
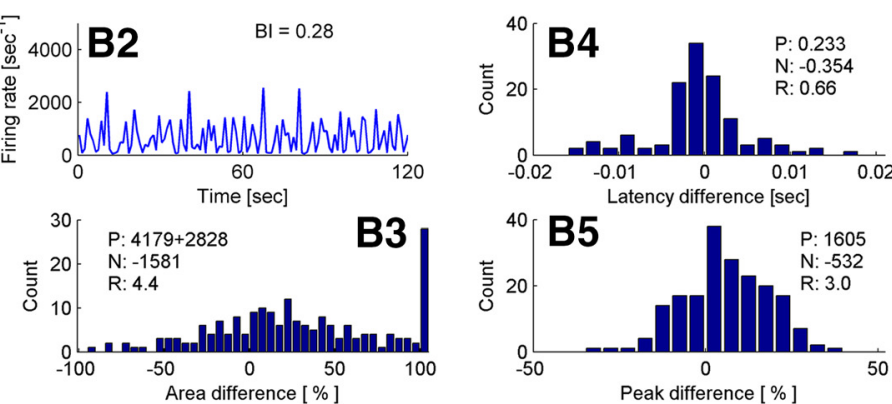

$\mathrm{C2}$
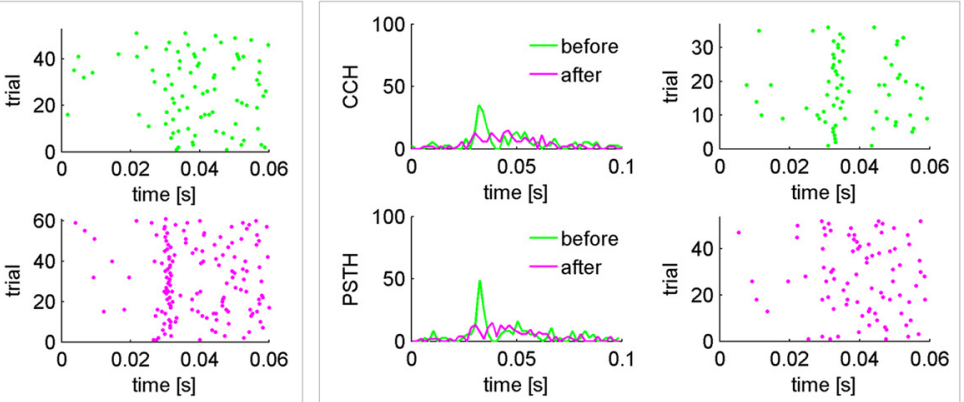

Figure 3. High-frequency tetanization induces LTD or LTP depending on culture "burstiness." A1-A5, Network connectivity change in a network with frequent SNBs. A1, Potentiated (red) and de-potentiated (blue) connections in the culture detected by CCH probings conducted right before and $5 \mathrm{~min}$ after $50 \mathrm{-Hz}$ stimulation (whole field stimulation, $50 \mathrm{~Hz}$ for 60 trials with 9-s intervals and 1-s stimulation time). A2, Network firing rate as a function of time, binning at $1 \mathrm{~s}$. BI (see Materials and Methods) is 0.83 , based on a 5min recording of network spontaneous activity, conducted before any stimulation. A3, Summary of efficacy change for all connections detected (see Materials and Methods, $\mathrm{CCH}$ probing, for more details regarding the calculation). $\mathrm{P}: \sum(0<\mathrm{changes} \leq 100 \%+$ changes $>100 \%$ ). $\mathrm{N}$ : $\sum$ (changes $<0 \%$ ). The unit of $\mathrm{P}$ and $\mathrm{N}$ is $[\%]$. $\mathrm{R}$ reflects the overall change in network efficacy $(\mathrm{R}=\mathrm{P} /|\mathrm{N}|)$. $\boldsymbol{A 4}$, Summary of the change in response latency for all connections detected. A5, Summary of the change in PSTH peak heights for all connections detected. B1-B5, Network connectivity change in a network with sparse SNBs (same format as $\boldsymbol{A 1 - A 5 ) . ~ C 1 , ~ C 2 , ~ C C H , ~}$ PSTH curves, and raster plots of the highlighted connections in $\mathbf{B 1}$ before (green) and after (magenta) the 50-Hz stimulation. $\mathbf{C 1}$ corresponds to connection $\mathrm{C} 1$, which was potentiated. The relative change of area under PSTH curve is $46.71 \%$. C2 corresponds to connection c2, which was depotentiated. The relative change of area under the PSTH curve is $-20.06 \%$. CCH curves were used to identify a connection and its response latency. PSTH curves were used to calculate the efficacy change.

$3 A 1-A 5]$. The decrease is quantified by a histogram of PSTH area relative changes centered at $-20 \%$ (for an example, see Fig. 3A3). On the contrary, in cultures where SNBs occurred less often with more asynchronous firings in the background, overall LTP was observed $[R=1.73 \pm 0.18$ (SEM), $n=3$; for an example, see Fig. 3B1-B5]. Figure 3C, panels 1,2 , provides examples for potentiated and depotentiated connections and the corresponding change in their $\mathrm{CCH}, \mathrm{PSTH}$ curves and raster plots. The protocol used in generating the results is $\mathrm{CCH}-50-\mathrm{Hz}$ tetanus $\mathrm{CCH}$.
In a further analysis examining the directionality of these connections, we noticed that the potentiated connections induced by $50-\mathrm{Hz}$ tetanization clustered around postsynaptic neurons (electrodes) rather than presynaptic neurons (grid positions; for an example, see Fig. 3B1). In our experiment setup, if the hub is an electrode, it will have only incoming connections; if it is a grid position, it will have only outgoing connections.

Comparing the electrode responses to SNBs and $50-\mathrm{Hz}$ tetani, we noticed that spontaneous firing patterns at individual electrodes during an SNB were more concentrated 
A

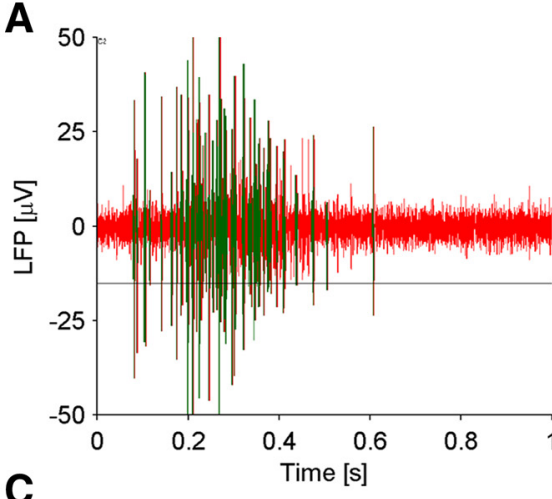

C

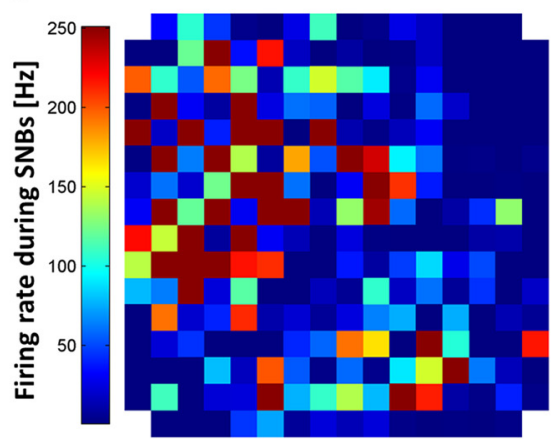

$E$

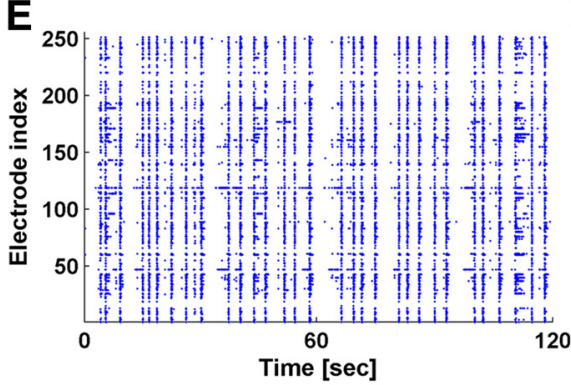

G

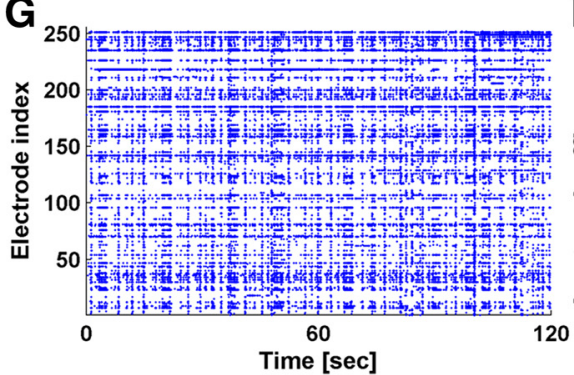

B
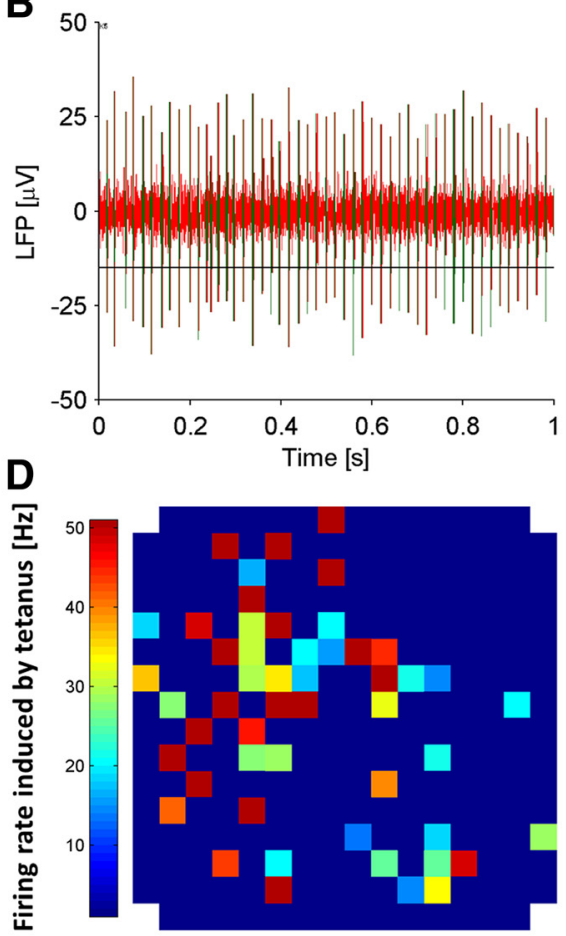

$F$

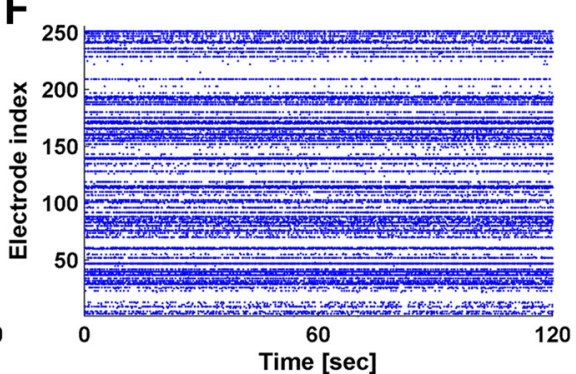

H

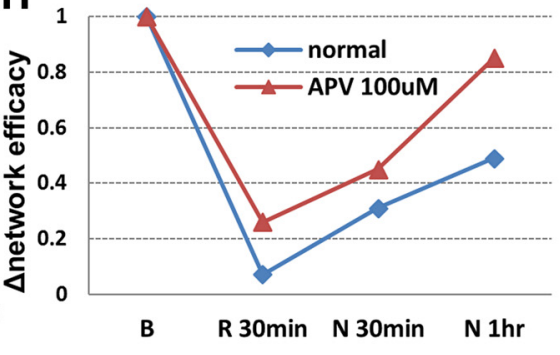

Figure 4. $\boldsymbol{A}-\boldsymbol{D}$, Comparison of network firing rate during SNBs and in response to $50-\mathrm{Hz}$ stimulation. $\boldsymbol{A}$, Local field potential (LFP) recorded at a single MEA electrode during an SNB. The red trace is noise and the green trace is when LFP exceeds the detection threshold set at $-6 \sigma$ of the noise. $\boldsymbol{B}$, LFP recorded at a single electrode during $50-\mathrm{Hz}$ light stimulation. $\boldsymbol{C}$, Network firing rate map during SNBs. Average firing rate was calculated for each electrode over 100-ms time bins during a 5-min recording of network activity with no external input. Firing rate in the top $15 \%$ bins was averaged to represent the firing rate during SNBs. No spike sorting was conducted. $\boldsymbol{D}$, Network firing rate map during response to whole-field $50-\mathrm{Hz}$ tetanization, averaged over 60 trials. Electrode signals collected from multiple neurons were filtered through spike sorting and the signal from the most synchronized neuron was retained for plotting. Notice the difference in color bar scales in $\boldsymbol{C}, \boldsymbol{D}$. $\boldsymbol{E}-\boldsymbol{G}$, raster plots of network activity in resting state $(\boldsymbol{E})$, in response to RDMs $(\boldsymbol{F})$, and resting state of a less synchronized culture $(\boldsymbol{G})$. $\boldsymbol{H}$, Network efficacy change ratio $[R=P /|N| ;$ see $M a t e r i a l s$ and Methods, $\mathrm{CCH}$ probing, for more details regarding the calculation] from baseline $(\boldsymbol{B})$ to 30 min under RDMs (R 30 min) followed by $30 \mathrm{~min}$ in resting state ( $\mathrm{N} 30 \mathrm{~min})$ and $1 \mathrm{~h}$ in resting state $(\mathrm{N} 1 \mathrm{~h})$. APV was added to the culture medium (100 $\mu \mathrm{m})$ to evaluate the contribution of NMDA receptors. 
with shorter duration $(0.2-0.3 \times)$ and larger amplitude $(1.5-2 \times)$, compared with induced response by $50-\mathrm{Hz}$ stimulation (Fig. 4A,B). This kind of ultrahigh-frequency spontaneous firing was observed at a large number of electrodes during an SNB (number of electrodes detected at least $50 \mathrm{~Hz}$ firing $>30$; Fig. $4 C$ ), whereas the number of electrodes detected synchronous firing to $50 \mathrm{~Hz}$ was limited (number of electrodes detected at least $50 \mathrm{~Hz}$ firing $\leq 17$; Fig. $4 D$ ).

\section{RDMs induce LTD prior to learning}

Given the analysis above, the question we addressed next is whether SNBs can be suppressed in cultures, while maintaining network viability. Pharmacological and electrical treatments have been proposed on this matter. Pharmacological reagents, such as NMDA receptors antagonist (e.g., APV and $\mathrm{Mg}^{2+}$ ) and gap junction blockers (e.g., carbenoxolone), are able to suppress SNBs to a great extent, but at the same time they also compromise the neuronal network functionality, especially its learning ability. A non-pharmacological method, i.e., randomly applied electrical stimuli through electrodes at high frequency, was suggested to quiet SNBs by mimicking the random noise coming from the external world (Wagenaar et al., 2005). Applying the quieting protocol resulted in enhanced functional plasticity (Madhavan et al., 2006) and increased likelihood of evoked network response (Goel and Buonomano, 2013). We therefore applied optogenetic stimulation in the form of RDMs (see Materials and Methods) to the neuronal networks prior to any learning stimulation, in an attempt to mimicking the effect of sensory inputs on the neocortex in an awake animal. As the cultures were extensively engaged in randomly induced firings during RDMs, SNBs emerged less frequently $[\mathrm{BI}=0.86 \pm 0.03$ (SEM), $n=6$ (for an example of culture activity before applying RDMs, see Fig. $4 E$ ); $\mathrm{BI}=0.09 \pm$ 0.02 (SEM), $n=6$; for an example of culture activity during RDM stimulation, see Fig. 4F)]. The activity of a less bursting culture seems to fall between the two extreme conditions $[\mathrm{BI}=0.38 \pm 0.08$ (SEM), $n=3$; for an example of less bursting cultures, see Fig. 4G].

We repeated the RDM stimulation protocol in the presence of $100 \mu \mathrm{m}$ APV in the culture medium to block NMDA receptors and compared the network efficacy changes observed with those seen in control cultures in the absence of APV. A reduced degree of depression immediately after a 30-min RDM and faster recovery after $1 \mathrm{~h}$ in resting state were observed when NMDA receptors were blocked (for an example, see Fig. 4H). The difference is marked by a network efficacy drop $<80 \%(R=0.53 \pm$ $0.27, n=2)$ in APV versus a network efficacy drop $>90 \%$ $(\mathrm{R}=0.07 \pm 0.004, n=3)$ without APV, and faster recovery to the original efficacy at $1 \mathrm{~h}(\mathrm{R}=1.67 \pm 0.82, n=2)$ after RDMs in APV versus prolonged depression at $1 \mathrm{~h}$ without APV $(R=0.36 \pm 0.08, n=3)$. The observed depression is unlikely to be a result of neurons dying, as the cultures survived well for days after the RDM stimulation. The decrease in efficacy in presence of APV is more likely a result of temporary network exhaustion or an NMDA-independent form of LTD (Pöschel and Manahan-Vaughan, 2005).
In contrast, the prolonged depression at $1 \mathrm{~h}$ after RDM stimulation in the absence of APV, suggests that LTD was induced and the process can be NMDAR dependent. Furthermore, the $\mathrm{CCH}$ probings conducted $5 \mathrm{~min}$ after RDM stimulation reveals network-wide LTD $(R=0.097 \pm$ $0.015, n=5$; for an example, see Fig. 5A). RDM-induced network-wide LTD potentially opens a window for enhanced learning, by creating more space for synaptic potentiation. The protocol used in generating the results above is $\mathrm{CCH}-\mathrm{RDM}-\mathrm{CCH}$.

\section{Learning specificity emerged after RDM pre- stimulation}

After the cultures were pre-stimulated with RDMs for at least $30 \mathrm{~min}$, we applied the same $50-\mathrm{Hz}$ stimulation protocol. $\mathrm{CCH}$ probing results show that, in contrast to the previous LTD outcome in cultures dominated by SNBs, network average efficacy change became LTP $(R>1$; Fig. $5 B$ ), similar to the LTP induced in the SNB-compromised cultures. To further validate that the LTP was indeed induced by the $50-\mathrm{Hz}$ stimulation rather than culture self-recovery from RDM pre-stimulation, we probed network responses to the three complex patterns at the testing phase. As a proper control, network efficacy change caused by the testing (Fig. $5 \mathrm{C}$ ) was not as significant as $50-\mathrm{Hz}$ stimulation, which confirms the minimum disturbance caused in baseline and testing phases. Comparing the PSTH curves of network response to the three patterns at baseline and testing, we found a pattern-specific firing rate increase for the learning stimulus $(n=5$; for two examples, see Fig. $6 B$ ). A similar firing rate increase was not observed in the learning experiments without RDM pre-stimulation ( $n=5$; for one example, see Fig. $6 A$ ). A statistical summary of the network activity over trials during the baseline/testing phases for the three cultures shown in Figure $6 A, B$ is provided in Figure $6 C$. The protocol used in generating the results above is $\mathrm{CCH}-\mathrm{RDM}-$ $\mathrm{CCH}-50-\mathrm{Hz}$ tetanus $-\mathrm{CCH}$.

The pattern-specific firing rate increase indicates that the neuronal networks have acquired familiarity to the trained pattern. Therefore, we conclude that learning after RDMs is more efficient than learning without RDM prestimulation. The ability to recognize familiar patterns seems to be an emergent and intrinsic network property, in which information of the complex patterns is separated in a high-dimensional feature space. PSTH curves at individual electrode channels evoked by the three patterns were compared before and after learning (Fig. 7A,B). Firing rate increase to the familiar pattern was observed at many channels widely distributed in the network.

The experimental paradigm was therefore updated from "baseline $\rightarrow$ learning $\rightarrow$ testing" (protocol is $\mathrm{CCH}-$ baseline $-\mathrm{CCH}-50-\mathrm{Hz}$ tetanus $-\mathrm{CCH}-$ testing $-\mathrm{CCH}$, referred as the conventional learning paradigm from here on) to "baseline $\rightarrow$ RDM $\rightarrow$ learning $\rightarrow$ testing" (protocol is $\mathrm{CCH}$ - baseline - $\mathrm{CCH}-\mathrm{RDM}-\mathrm{CCH}-50 \mathrm{~Hz}-\mathrm{CCH}-$ testing $-\mathrm{CCH}$, referred as the RDM-learning paradigm from here on). Baseline recording was conducted before RDM pre-stimulation to prevent unwanted network recovery induced by the baseline recording. This way, the 


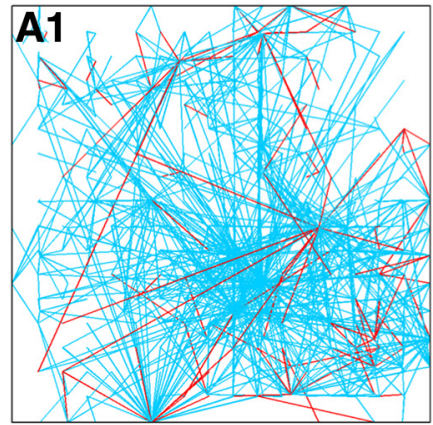

CCH-RDM-CCH

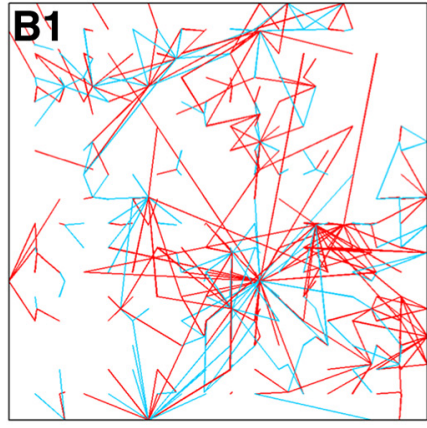

$\mathrm{CCH}-50 \mathrm{~Hz}$ tetanus- $\mathrm{CCH}$

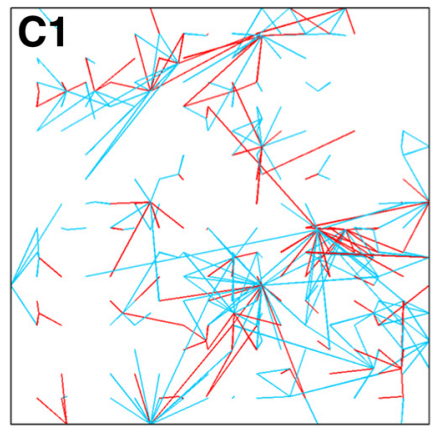

$\mathrm{CCH}$-testing- $\mathrm{CCH}$
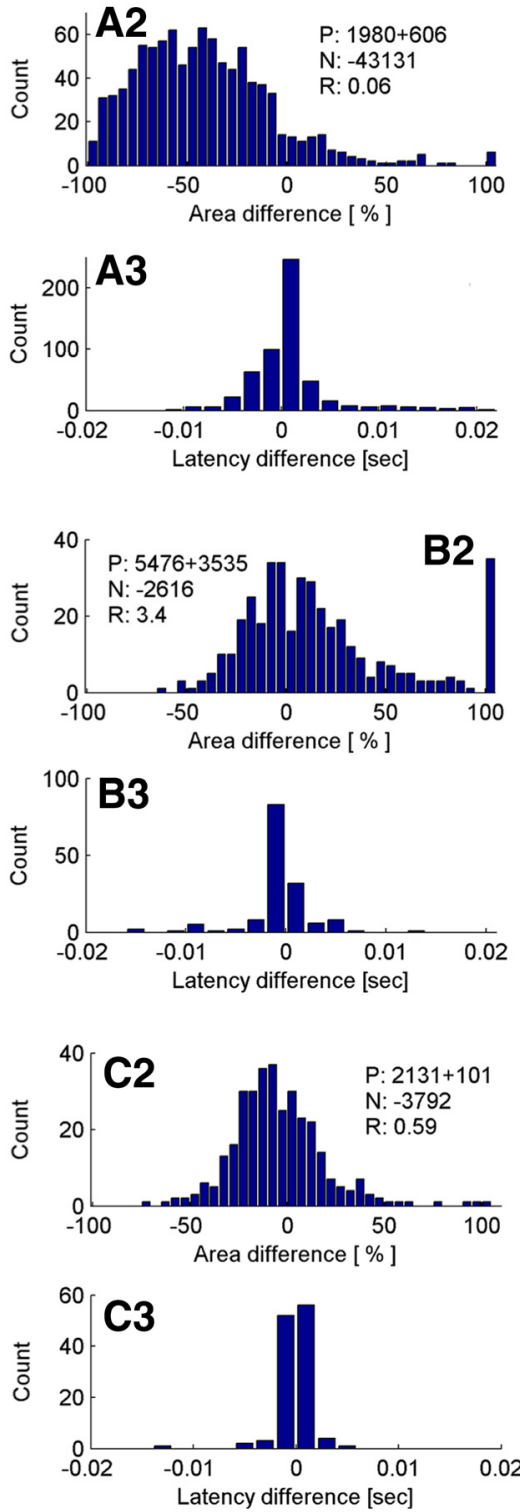

Figure 5. RDM-induced network-wide LTD enables future LTP. A1-A3, Network connectivity changes induced by 30 min RDM stimulation. A1, Potentiated (red) and depotentiated (blue) connections in the culture detected by two CCH probings conducted immediately before and $5 \mathrm{~min}$ after the 30-min RDMs. A2, Summary of the relative change in area under the PSTH curves for all the connections detected. See Materials and Methods for the meanings of $\mathrm{P}, \mathrm{N}$, and $\mathrm{R}$ numbers. A3, Summary of the change in response latency for all the connections detected. B1-B3, Network connectivity changes induced by $50-\mathrm{Hz}$ stimulation conducted after the RDMs (same format as $\boldsymbol{A 1 - A 3 ) . ~ T h e ~ h u m a n ~ f a c e ~ p a t t e r n ~ w a s ~ u s e d ~ a s ~ t h e ~ l e a r n i n g ~ s t i m u l u s . ~ C 1 - C 3 , ~ N e t w o r k ~ c o n n e c t i v i t y ~}$ changes caused by the testing recording (same format as $\boldsymbol{A 1}-\boldsymbol{A} 3$ ).

network baseline responses were generally higher than the testing responses (Fig. 6B2), because RDMs induced significant network LTD, and the subsequent learning only selectively potentiated a subset of the connections.

\section{Memory consolidation in vitro}

What we have observed so far is at best early-phase LTP (Huang, 1998; Blundon and Zakharenko, 2008). During the experiments, $\mathrm{CCH}$ probings were conducted $5 \mathrm{~min}$ after learning, and network responses to the three patterns were obtained $10 \mathrm{~min}$ after learning at the testing phase. This time scale is longer than short-term memory but still short for long-term memory. Given that SNBs occurred sporadically in the background, which might result in an ongoing modification of network connectivity, we wanted to address the question whether the encoded familiarity would be consolidated into long-term memory.

In intact animals, memory consolidation has been correlated with high-frequency oscillations that can be observed during sleep. Cultures with SNBs have been proposed as a slow-wave sleep model (Colombi et al., 2016). Therefore, it is reasonable to hypothesize that SNBs may be retaining the encoded pattern by spontaneously initiating neuronal activities through the potentiated 
A
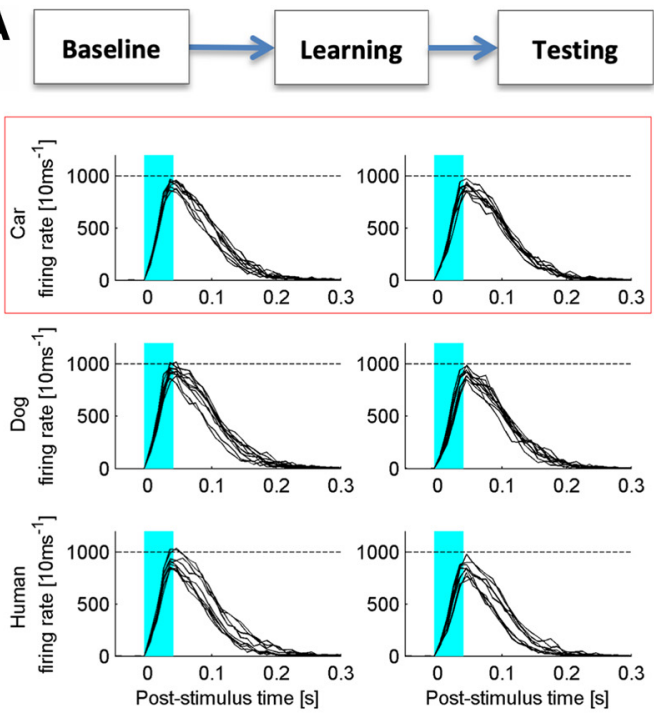

C
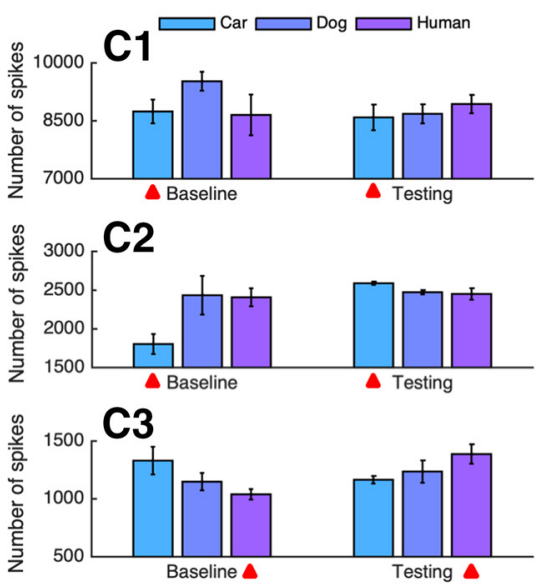

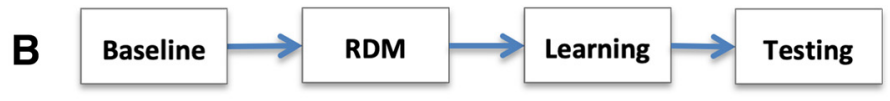

B1
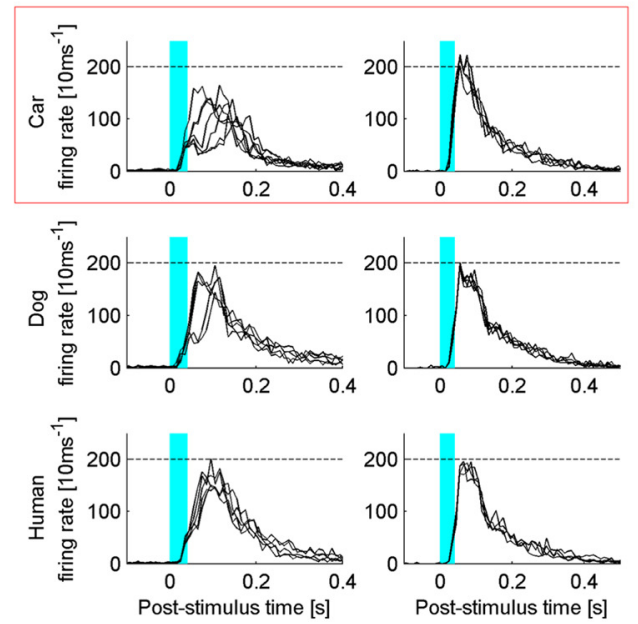

\section{B2}
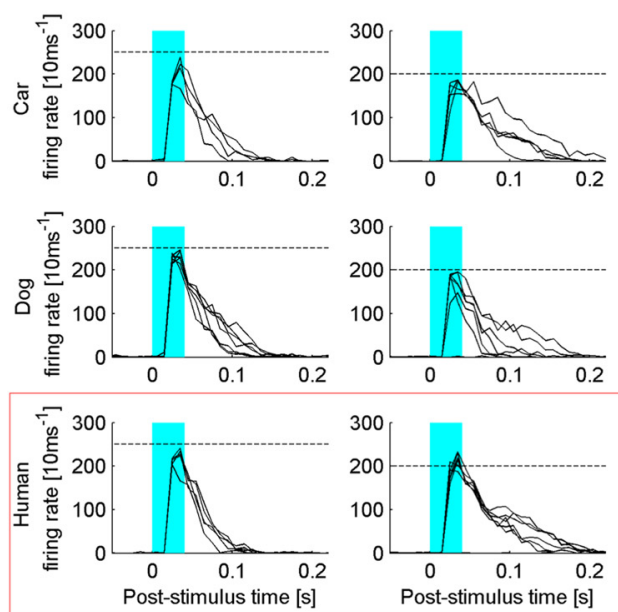

Figure 6. PSTHs of the network responses to the three complex patterns probed at baseline and testing phases. Noisy trials in which SNBs occurred within $1 \mathrm{~s}$ before the pattern stimulus onset were removed. Blue indicates the stimulus duration. Red boxes indicate the patterns presented during the learning phase. The left column is baseline response. The right column is testing response. A, Baseline and testing responses recorded from an MEA culture with non-RDM learning. B, Baseline and testing responses recorded from two MEA cultures with learning after RDM pre-stimulation. Dotted lines were drawn at the same heights for all three patterns at baseline/testing to aid visualization of firing rate difference. $\boldsymbol{C}$, Summarizing the average number of spikes (mean area under PSTH curves + SEM) induced by each pattern over trials during baseline/testing phases for the three cultures shown in $\boldsymbol{A}, \boldsymbol{B}$. $\boldsymbol{C} 1$ corresponds to $\boldsymbol{A} ; \boldsymbol{C 2}$ corresponds to $\mathbf{B 1} ; \boldsymbol{C} \mathbf{3}$ corresponds to $\mathbf{B 2}$. Red arrows indicate the patterns presented during the learning phase.

connections. In order to decode the information contained in the SNBs, we used an SVM approach to classify SNBs.

At first, SVM classifiers were trained to recognize network responses induced by the three complex patterns. Then, the SVM classifier trained with pattern-induced responses obtained at the baseline phase was applied to classify SNBs in the 5-min continuous recording obtained before the baseline phase; the SVM classifier trained with pattern-induced responses obtained at the testing phase was applied to classify SNBs in the 5-min continuous recording obtained immediately after the learning phase. Classified labels of SNBs before and after learning are compared. The results show that after the RDM-learning paradigm, a larger portion of SNBs were classified as the trained pattern (Fig. 7D), indicating a higher similarity in the network activity pattern. In other words, SNBs carried more information of the trained pattern after learning. 

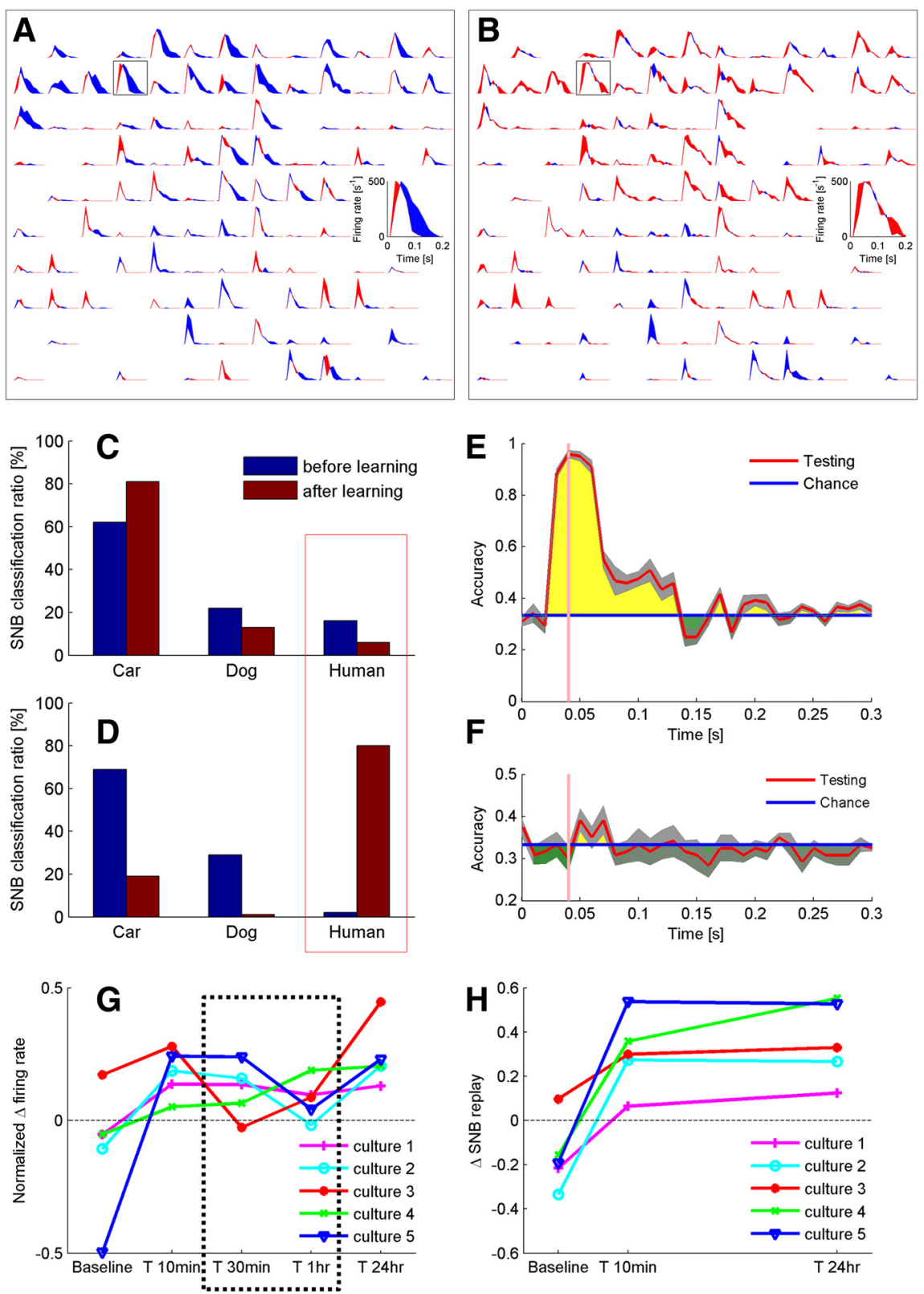

Figure 7. $\boldsymbol{A}, \boldsymbol{B}, \mathrm{PSTH}$ difference in response to the training and control patterns at individual electrodes. $\boldsymbol{A}$, Difference between the responses to a human face (trained) and a car front (control) stimulus pattern at baseline. Red indicates a higher PSTH to the human face and blue indicates a higher PSTH to the car front. PSTHs are summed over 20-ms bins. An average PSTH curve of 10 trials is calculated to represent the electrode response to the probing pattern. The boxed electrode response is enlarged as the inset; $x$-scale is [0, $0.2 \mathrm{~s}$ ] for all; $y$-scale is $[0,500]$ spikes/s for all. Only the most active portion of the electrode array is shown (10 $\times 13$ electrodes). Only the response to car front is shown as the response to a control pattern for clarity. $\boldsymbol{B}$, Difference between the responses to a human face (trained) and a car front (control) stimulus pattern at testing (same format as $\boldsymbol{A}$ ). $\boldsymbol{C}, \boldsymbol{D}$, SVM classified labels for SNBs recorded before (blue) and after learning (red) in the conventional learning paradigm $(\boldsymbol{C})$ and the RDM-learning paradigm (D). Five-minute continuous recordings of network spontaneous activity were used. Red box indicates the trained pattern. $\boldsymbol{E}, \boldsymbol{F}$, SVM classification accuracy on pattern-induced responses $(\boldsymbol{E})$ and after we randomized the pattern labels for training as a control $(\boldsymbol{F})$. The pink bar at $0.04 \mathrm{~s}$ indicates the termination of light stimulus. Gray shading indicates the SEM of 10 trained SVMs. Yellow indicates classification accuracy above chance level and green for below chance level. $\mathbf{G}, \boldsymbol{H}$, Memory consolidation in cultures with extended testing phase. G, Network response to the trained pattern was probed at $10 \mathrm{~min}, 30 \mathrm{~min}, 1 \mathrm{~h}$, and $24 \mathrm{~h}$ after the learning phase. Network firing rate induced by the image cues was used to evaluate memory recall. Firing rate discrepancies between the familiar pattern and the two control patterns were averaged and then the value was normalized with respect to the familiar pattern to make the results comparable among cultures (see Materials and Methods, Evaluation metric, for more details regarding the calculation). $\boldsymbol{H}$, SVM classification of SNBs recorded at baseline, $10 \mathrm{~min}$ and $24 \mathrm{~h}$ after learning was conducted by using the network responses obtained in $\boldsymbol{G}$ as training sets. SNB ratios (see $\boldsymbol{C}, \boldsymbol{D}$ for an example) between the familiar pattern and the two control patterns were averaged to make the results comparable among cultures (see Materials and Methods, Evaluation metric, for more details regarding the calculation). Same cultures as in G. 
Therefore, the trained pattern is likely being replayed during SNBs, potentially undergoing memory consolidation. On the contrary, SNBs after the conventional learning paradigm did not resemble the trained pattern more (Fig. 7C). Separately, we validated the trained SVM classifiers by looking at their classification accuracy of an independent set of pattern-induced network responses $(60 \%$ data were used for training, $40 \%$ data for testing) recorded at baseline. The results show good accuracy at $30-120 \mathrm{~ms}$, while the pattern stimuli were delivered at $0-40 \mathrm{~ms}$ (Fig. $7 E$ ). When the pattern labels were randomized prior to SVM training, the classification accuracy reduced to chance level (Fig. 7F).

To further investigate the long-term retention of the encoded familiarity, we extended the testing phase in an independent set of experiments by including three sessions of testing spaced at $30 \mathrm{~min}, 1 \mathrm{~h}$, and $24 \mathrm{~h}$ after the learning phase in addition to the original testing conducted at $10 \mathrm{~min}$. While network firing rate distinction was observed to be not well maintained $30 \mathrm{~min}$ after learning, network firing rate to the familiar pattern re-appeared to be on top when probed at $24 \mathrm{~h}$ after learning (Fig. 7G). A similar effect has been reported by another group (Chiappalone et al., 2008, their Fig. S1), who found network response maintenance $1 \mathrm{~d}$ after LTP induction experiments. So, if we only focus on the familiarity recall with 1 -d retention (24 h), the network firing rate discrepancy between familiar and control patterns is as good as when recalled immediately after learning (10 min), implying the existence of memory consolidation. A similar trend was observed with the corresponding SNB replay ratio (Fig. $7 H$ ). The protocol used in generating the results above is $\mathrm{CCH}-\mathrm{RDM}-50$ $\mathrm{Hz}$ tetanus - $\mathrm{CCH}(10 \mathrm{~min})-\mathrm{CCH}(30 \mathrm{~min})-\mathrm{CCH}(1 \mathrm{~h})-$ $\mathrm{CCH}(24 \mathrm{~h})$.

\section{Comparison between conventional learning and RDM- learning paradigms}

Summarizing all the findings above, we present here the major difference between the conventional learning paradigm and the novel RDM-learning paradigm. As the dynamics of in vitro dissociated neuronal networks are highly variable and noisy in nature, we have had plenty of outlier cultures with unique behavior that could not be subsequently reproduced. This paper therefore focuses on the most reproducible observations. When $50-\mathrm{Hz}$ tetanus was given to cultures during the learning phase, little LTP $(R<2)$ was observed following the conventional learning paradigm (Fig. 8A), whereas significant LTP $(R>2)$ was observed following the RDM-learning paradigm (Fig. 8B). One prerequisite for the training to be efficient in the RDM-learning paradigm was that the RDM pre-stimulation had to induce major depression in the network efficacy $[R=0.097 \pm 0.015$ (SEM), $n=5$; Fig. $8 B]$. In cultures that RDM pre-stimulation did not induce sufficient depression, the subsequent firing rate distinction was compromised. In the conventional learning paradigm, network firing rate to the trained pattern was decreased in general (Fig. 8C), suggesting mild LTD induction following the $50-\mathrm{Hz}$ tetanization. Although pattern-specific LTD might be an alternative learning outcome, the encoded LTD is unlikely to be replayed by the background SNBs (Fig. 8E) and therefore will eventually be lost over time. In the RDM-learning paradigm, network response to the three patterns was first greatly suppressed by the RDMs and then a subset of neurons was selectively stimulated and recruited to store the encoded familiarity, resulting in a comparatively higher network firing rate to the trained pattern among the three (Fig. 8D). More importantly, postencoding SNBs spontaneously reactivate the memory engram (Fig. $8 F$ ), which provides means to maintain the strengthened pathways in the network and potentially creates a robust model for learning and memory studies in vitro.

\section{Discussion}

The goal of the experiments described above was to study learning and memory in neuronal networks. A major issue holding back progress in networks formed by cultured cortical neurons is the spontaneous occurrence of highly SNBs.

\section{Development of SNBs}

SNB activity patterns change with the developmental stage of cultured cortical neurons, and they have been shown to disrupt the encoding of short-term memory (Dranias et al., 2015; Ju et al., 2015). In addition, it has been postulated that SNBs are essential for neuronal network development and maturation (Luhmann et al., 2016). The fact that properly maintained MEA cultures can survive for months (Potter and DeMarse, 2001) creates an opportunity to study the development of the functional activity of these neuronal networks in detail. In our experiments, SNBs emerged as early as DIV8, indicating the existence of global connections at this early age, However, long-range connections were not detectable by our $\mathrm{CCH}$ analysis until the culture had adequately matured. The sparsity of detectable global connections before DIV22 is likely the result of synaptic efficacies being relatively weak initially. Normally, spatial and temporal summation of multiple inputs is required to trigger a postsynaptic action potential. In order to be detected by $\mathrm{CCH}$ probing, a global connection must be strong enough to trigger the firing of the postsynaptic neuron by itself. The long-range connections that were uncovered in the connectivity map at DIV19 (Fig. 2E) by lowering the $\mathrm{CCH}$ detection threshold possibly represent newly formed connections which are still relatively weak. Some of these can strengthen as the network matures. In other words, the $\mathrm{CCH}$ analysis relies on strong connections to reconstruct the network architecture. We have focused on such strong connections, because they are more stable and reliable when we look for subtle changes due to plastic modification.

\section{Long-term effect of SNBs}

In cultures dominated by SNBs, high frequency stimulation with a training pattern caused an LTD of network activity (Fig. 3A1-A5). The overall LTD is consistent with observations reported earlier (Chiappalone et al., 2008), that high-frequency tetanization caused an overall decrease in 

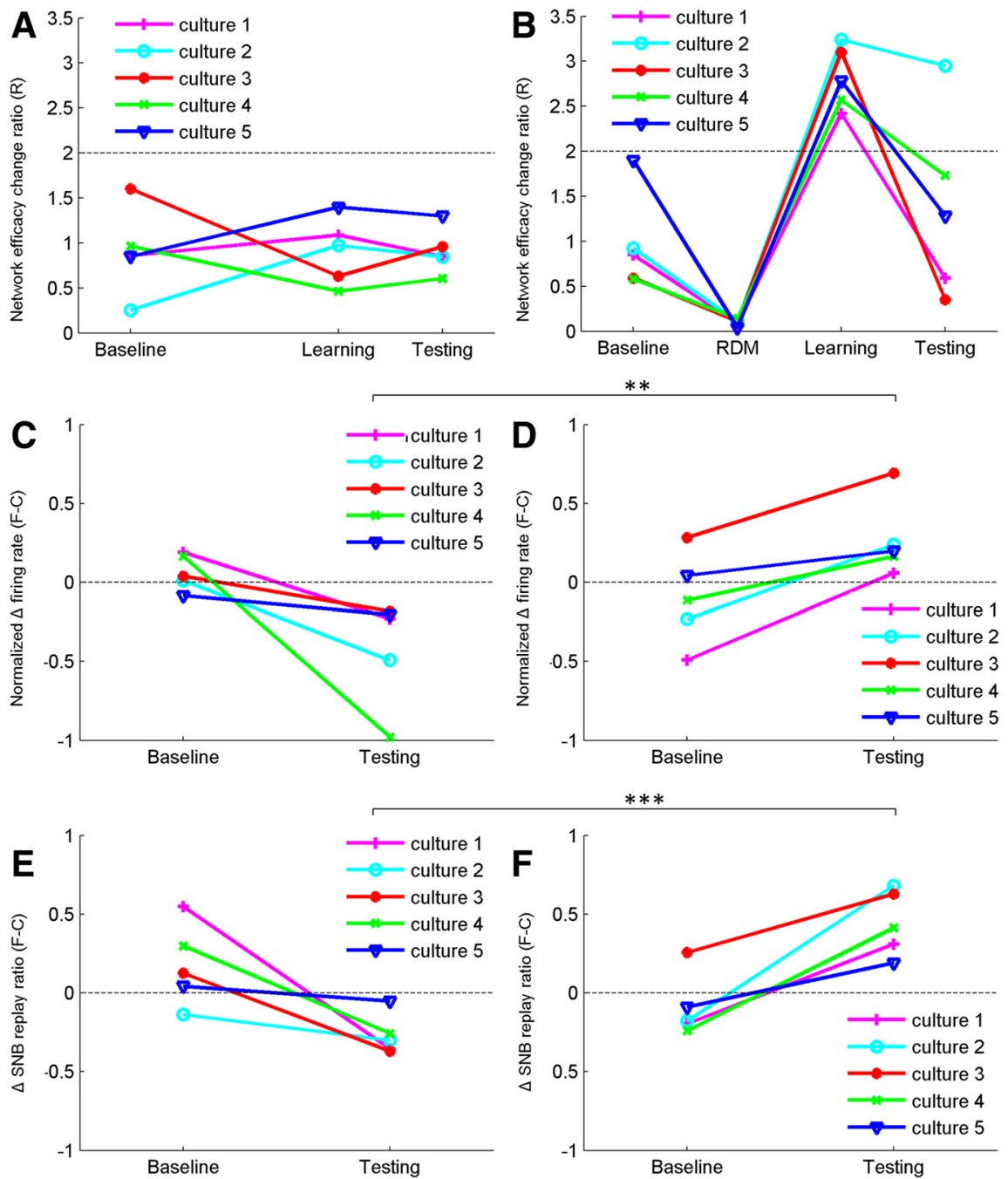

Figure 8. Summary of culture performance under the conventional learning paradigm $(\boldsymbol{A}, \boldsymbol{C}, \boldsymbol{E})$ and the RDM-learning paradigm (B, $\boldsymbol{D}, \boldsymbol{F}) . \boldsymbol{A}, \boldsymbol{B}$, Network efficacy change ratio $[\mathrm{R}=\mathrm{P} /|\mathrm{N}|$; see Materials and Methods, $\mathrm{CCH}$ probing, for more details regarding the calculation] induced at the baseline phase, RDM pre-stimulation, learning phase, and testing phase for experiments conducted under the conventional learning paradigm $(\boldsymbol{A})$ and the novel RDM-learning paradigm $(\boldsymbol{B})$. $\boldsymbol{C}, \boldsymbol{D}$, Network firing rate discrepancy between familiar and control patterns (see Materials and Methods, Evaluation metric, for more details regarding the calculation; $p<0.01\left({ }^{* *}\right)$ for unpaired two-sample $t$ test) for experiments conducted under the conventional learning paradigm (C) and the novel RDM-learning paradigm $(\boldsymbol{D})$. $\boldsymbol{E}, \boldsymbol{F}$, Change in SNB replay ratio for the familiar pattern (see Materials and Methods, Evaluation metric, for more details regarding the calculation; $\left.p<0.001{ }^{* \star *}\right)$ for unpaired two-sample $t$ test) for experiments conducted under the conventional learning paradigm $(\boldsymbol{E})$ and the novel RDM-learning paradigm $(\boldsymbol{F})$. Different sets of cultures were used in each panel.

evoked network response, rather than a global increase as conventionally expected. For cultures in which SNBs were sparse and which displayed more asynchronous firing, tetanization with a pattern resulted in LTP (Fig. 3B1-B5). Potentiated connections clustered around a few postsynaptic neurons (Fig. 3B1). This result supports the idea that these postsynaptic hub neurons, receiving multiple inputs from concurrently stimulated dot positions of the training pattern, have a higher chance to fire synchronously in response to high-frequency stimuli, which in turn results in potentiation of the synapses between them and their presynaptic inputs following the "Hebbian" rule.

An individual MEA electrode may record from between one and four neurons (Litke et al., 2004). Therefore, network firing frequency recorded by the MEA during SNBs can be much higher than the $50-\mathrm{Hz}$ frequency we used for tetanization. It is reasonable to assume that the neuronal networks have been excessively potentiated by SNBs, leaving little space for future LTP. In other words, SNBs impose a higher LTP threshold for in vitro neuronal networks.

SNBs have been hypothesized to assist the synaptic development and maturation of the neuronal networks in young cultures (Kerschensteiner, 2014). However, the results described above potentially implicate the adverse long-term effect of having SNBs in cultures.

\section{Learning familiar inputs after RDMs}

We have shown that stimulating the networks with RMDs induces a form of LTD, in which the frequency of 
SNBs is substantially reduced. We have used a BI to monitor this process. If we view the baseline network activity with abundant SNBs as purely ordered (for an example, see Fig. 4E) and activity during RDMs as purely disordered (for an example, see Fig. 4F), then the activity of the networks after RMD treatment (for an example, see Fig. 4G) seems to fall at the "edge of chaos," which has been predicted to have optimal computational power (Bertschinger and Natschläger, 2004; Legenstein and Maass, 2007).

High-frequency tetanization after RDM pre-stimulation induced pattern-specific LTP. The pattern-specific network firing rate increase indicates that the neuronal networks have acquired familiarity to the trained pattern. Therefore, we conclude that the ability to recognize familiar complex patterns is an emergent and intrinsic property of neuronal networks that are maintained in vitro. The network's ability to discern patterns and recognize a familiar input has been predicted by previous studies with simulated neural network models. Constrained by the spatial resolution of MEAs, we could not measure the activity of each individual neuron in the network, and therefore we could not identify the critical neurons and subnetworks responsible for familiarity, as was done for the simulated neural networks. Nevertheless, based on the PSTH curves of network responses at individual electrodes, we can tell that the firing rate increase to the familiar pattern is distributed to many channels in the network, implying it reflects a network property. Similar observation has been found in reservoir computing (Subramoney et al., 2019).

In the learning experiments, after we applied patternspecific optogenetic stimulation, we probed the network responses to both trained and control patterns. By including control patterns, it came to our attention that in cultures dominated by periodic SNBs, high-frequency tetanization induced little learning specificity. It was only after we prestimulated the cultures with RDMs which counteracted the SNBs by reducing network efficacy on average, we started seeing a difference in evoked network responses induced by high-frequency tetanization between the training and control patterns (Fig. 6). By showing that the network response change is directly associated with the trained pattern, we established a more specific causal relationship that is less likely to be confounded by fluctuations in network background activity. Missing proper controls and the lack of direct causality in the early studies prior to Wagenaar et al. (2006b) potentially undermine their reliability, because changes in the network firing rate, SNB frequency or response latency are not exclusive to learning but could simply be caused by fluctuations and drifts in network excitability.

The flexibility provided by optogenetic stimulation and the novel $\mathrm{CCH}$ probing method allows us to examine changes in the network at each step and provides more insight into the functional role of SNBs. For cultures without RDM pre-stimulation, high-frequency tetanization induced LTD. Whereas for cultures after RDM pre-stimulation or cultures with naturally compromised SNBs, high-frequency tetanization induced LTP. Applying CCH probings, we discovered that RDM pre-stimulation induces a network-wide depression. These phenomena together suggest chronic
SNBs have excessively potentiated the networks, leaving little space for future potentiation (Wagenaar et al., 2006b). Therefore, when high frequency tetani were delivered, the networks underwent changes skewed to the LTD side, impairing the learning specificity. We suspect that the equivocal observations from previous MEA studies stem from differences in network SNB levels at the resting state. Many of the aforementioned studies did not characterize the bursting status of their cultures, leaving the problem open for further investigation.

For experiments performed using the RMD-learning pathway, the network baseline responses were generally higher than the testing responses (Fig. 6B2), because RDMs induced significant network LTD, and the subsequent learning only selectively potentiated a subset of the connections. Nevertheless, occasionally we observed the opposite, namely that the testing responses were higher in firing rate than baseline responses (Fig. 6B1). This occurred when there was an increase in average IBI duration for SNBs in the background from baseline to testing, suggesting that the networks were more engaged in SNBs at baseline, but more entrained to external stimuli at testing. This phenomenon can happen in either paradigm, i.e., learning with or without RDM pre-stimulation. When it occurred in the conventional learning paradigm, despite the overall firing rate increase in evoked network responses at the testing phase, no learning specificity to the trained pattern was co-observed, indicating the change was not correlated with familiarity encoding. This phenomenon is easily confused with a real learning effect when there is no rigorous control.

Based on our experience, a sufficiently dense, viable MEA culture will be dominated by SNBs from DIV13 onwards. Although the bursting has been regarded as seizure-like in some studies (Wagenaar et al., 2005; Ahn et al., 2017), it is more likely to be a result of the absence of external inputs during the development of neuronal networks (Madhavan et al., 2006). The frequency of SNBs in a mature culture stabilizes at $0.1-0.2 \mathrm{~Hz}$, which falls into the frequency range of the $\delta$ brain waves detected by EEGs $(0.1-4 \mathrm{~Hz}) . \delta$ Waves are characterized as the slowest brain waves with the highest amplitude and are commonly found in stage N3 slow-wave sleep (Vlahou et al., 2014). Therefore, a few recent studies suggest that the emergence of SNBs signifies MEA cultures in a state equivalent to chronic sleep. Similarity in gene expression profiles between cortical cultures and sleep models provides further support for this notion (Hinard et al., 2012). Carbachol, a cholinergic agonist, was applied to the cultures and successfully transformed the network activity from resembling slow-wave sleep to more like activity during rapid eye movement (REM) sleep (Colombi et al., 2016). We noticed that the network activity induced by RDM pre-stimulation (Fig. 4F) showed a similar pattern to the Carbachol-induced REM-like activity. The fact that RDM pre-stimulation counteracted SNBs and caused network-wide depression may be suggesting how synaptic efficacy homeostasis is established in the brain.

A few studies investigating learning and memory in cultured neuronal networks attempted to circumvent the SNB obstacles. One group was able to show the 
networks' ability for pattern separation with L-shape patterns, but they argued that electrical tetanization with a frequency above $200 \mathrm{~Hz}$ was required to observe the learning specificity (Ruaro et al., 2005). It seems that using a higher frequency may be able to conquer the over-potentiation problem caused by SNBs. Nevertheless, inducing such an ultrahigh-frequency tetanus is not feasible with our optogenetic stimulation. The membrane properties of cortical neurons prevent them from firing synchronously faster than $50 \mathrm{~Hz}$. Therefore, it is impossible to induce $200-\mathrm{Hz}$ spiking activity in a physiological condition. Another group demonstrated learning specificity in networks' direct response to stimuli after pharmacologically inducing neurogenesis (Tanaka et al., 2017), which potentially introduced more immature synapses to the network whose efficacy had not yet been over-potentiated by SNBs. In a study that failed to induce network LTP with high-frequency tetanization (Chiappalone et al., 2008), it was shown that associative memories could be induced in the cultured networks by pairing a session of high-frequency tetanus with an inphase single pulse applied at a spatially distant electrode. The mechanism underlying the in-phase pairing protocol for associative memory induction was not explicitly explained. In a following review article, it was speculated that the in-phase pairing protocol induced stronger network bursts than a session of high-frequency tetanus alone (Bologna et al., 2010). In addition, metaplasticity has been reported underlying associative memory (Sharma and Sajikumar, 2014; Xu et al., 2014), and the in-phase pairing protocol might be an efficient way of modifying metaplasticity in the cultured networks, which in turn modifies the threshold for synaptic plasticity induction.

SNBs re-appear after the RDM-learning-testing sequence, and $24 \mathrm{~h}$ later they still encode information specific to the learned pattern (Fig. 7), suggesting that they take part in memory consolidation. Regarding why the network firing rate to the familiar pattern was not consistently the highest for the shorter retentions $(30 \mathrm{~min}$ and 1 h), we do not have an explicit answer for now. It has been shown that postencoding maintenance of the activity pattern present during learning is critical for memory consolidation (Guzman-Ramos and Bermudez-Rattoni, 2011; Park et al., 2016). Specifically, a time course study that investigated the influence of transient inactivation of perirhinal cortex on recognition memory (Winters and Bussey, 2005) showed that memory consolidation was impaired when the cortical neuron activity was suppressed by lidocaine shortly after encoding (0-20 min), but no impairment was found if the suppression was incurred 40 min after encoding. It was then suggested that at some time point beyond 20 min after encoding, the memory trace became sufficiently strengthened to resist any disruption to the neuronal activity. On account of this, it is possible for us to observe the firing rate decay at 30-min and 1-h time points, while the memory was still consolidated at $24 \mathrm{~h}$. On the other hand, the weakened response to the familiar pattern at $30 \mathrm{~min}$ and $1 \mathrm{~h}$ might be a network strategy to get ready for new information processing (Oliveira et al., 2010) and prevent encoded memory being overwritten. Nevertheless, it is important to note that each of the cultures that we studied is a single network, which carries no hierarchical regulations like in the brain. We should take caution to avoid over-explaining the firing rate decay, as it may be simply due to network exhaustion after multiple testing sessions in a relatively short window.

The presence of SNBs may not be entirely a bad thing. In fact, spontaneous synchronized activity is common to the brain at many levels (Corlew et al., 2004; Blankenship and Feller, 2010). On the largest scale, spontaneous oscillations have been observed for the entire brain by $\mathrm{fMRI}$ and EEG (Burke et al., 2013). On the brain circuit scale, it has been shown that high-frequency synchronized activity in the hippocampal-cortical dialogue during slow-wave sleep is involved in memory consolidation (Mitra et al., 2016), reflected as sharp-wave ripples in hippocampus and sleep spindles/k-complexes in cortex (McVea et al., 2016). Neuronal activity patterns evoked during learning have been found to be replayed in the hippocampus during sleep (Breton and Robertson, 2013). There is a chance that SNBs in vitro function in a similar way to enforce the strengthened connections. The SVM classification results show that the encoded pattern was "replayed" by SNBs after learning. It sheds some light on the existence of memory consolidation mechanism in neuronal networks. Therefore, even in the presence of SNBs, in vitro neuronal networks can be used to study learning and memory with proper preparations.

\section{Conclusion}

In this study, a novel probing method was applied to scrutinize the network change in response to learning. By counteracting spontaneously occurring SNBs, we managed to induce pattern-specific familiarity, an observation that is comparable to what has been demonstrated in silico and has never been reported before for in vitro neuronal networks. The postlearning activity pattern of SNBs carried more information of the familiar stimulus, suggesting a mechanism of memory consolidation. It is concluded that in vitro neuronal networks can acquire familiarity to complex patterns with specificity, and we suggest a novel elucidation to the role of SNBs as a double-edged sword that disrupts the encoding of new memories and facilitates the consolidation of existing memories at the same time.

\section{References}

Ahn S, Jo S, Jun SB, Lee HW, Lee S (2017) Study on the mechanisms of seizure-like events suppression effect by electrical stimulation using a microelectrode array. Neuroreport 28:471-478.

Arenkiel BR, Peca J, Davison IG, Feliciano C, Deisseroth K, Augustine GJ, Ehlers MD, Feng G (2007) In vivo light-induced activation of neural circuitry in transgenic mice expressing channelrhodopsin-2. Neuron 54:205-218.

Bertschinger N, Natschläger T (2004) Real-time computation at the edge of chaos in recurrent neural networks. Neural Comput 16:1413-1436.

Blankenship AG, Feller MB (2010) Mechanisms underlying spontaneous patterned activity in developing neural circuits. Nat Rev Neurosci 11:18-29.

Blundon JA, Zakharenko SS (2008) Dissecting the components of long-term potentiation. Neuroscientist 14:598-608. 
Bologna LL, Nieus T, Tedesco M, Chiappalone M, Benfenati F, Martinoia S (2010) Low-frequency stimulation enhances burst activity in cortical cultures during development. Neuroscience 165:692-704.

Breton J, Robertson EM (2013) Memory processing: The critical role of neuronal replay during sleep. Curr Biol 23:R836-R838.

Burke JF, Zaghloul KA, Jacobs J, Williams RB, Sperling MR, Sharan $A D$, Kahana MJ (2013) Synchronous and asynchronous theta and gamma activity during episodic memory formation. J Neurosci 33:292-304.

Chang CC, Lin CJ (2011) LIBSVM: A library for support vector machines. ACM Trans Intell Syst Technol 2:1-27.

Chiappalone M, Novellino A, Vajda I, Vato A, Martinoia S, van Pelt J (2005) Burst detection algorithms for the analysis of spatio-temporal patterns in cortical networks of neurons. Neurocomputing 65:653-662

Chiappalone M, Vato A, Berdondini L, Koudelka-Hep M, Martinoia S (2007) Network dynamics and synchronous activity in cultured cortical neurons. Int J Neural Syst 17:87-103.

Chiappalone M, Massobrio P, Martinoia S (2008) Network plasticity in cortical assemblies. Eur J Neurosci 28:221-237.

Colombi I, Tinarelli F, Pasquale V, Tucci V, Chiappalone M (2016) A simplified in vitro experimental model encompasses the essential features of sleep. Front Neurosci 10:315.

Corlew R, Bosma MM, Moody WJ (2004) Spontaneous, synchronous electrical activity in neonatal mouse cortical neurones. J Physiol 560:377-390.

Dranias MR, Westover MB, Cash S, VanDongen AMJ (2015) Stimulus information stored in lasting active and hidden network states is destroyed by network bursts. Front Integr Neurosci 9:14.

Dudai $Y$ (1997) How big is human memory, or on being just useful enough. Learn Mem 3:341-365.

Eytan D, Marom S (2006) Dynamics and effective topology underlying synchronization in networks of cortical neurons. J Neurosci 26:8465-8476.

Goel A, Buonomano DV (2013) Chronic electrical stimulation homeostatically decreases spontaneous activity, but paradoxically increases evoked network activity. J Neurophysiol 109:1824-1836.

Gunaydin LA, Yizhar O, Berndt A, Sohal VS, Deisseroth K, Hegemann P (2010) Ultrafast optogenetic control. Nat Neurosci 13:387-392.

Guzman-Ramos K, Bermudez-Rattoni F (2011) Post-learning molecular reactivation underlies taste memory consolidation. Front Syst Neurosci 5:79.

Hinard V, Mikhail C, Pradervand S, Curie T, Houtkooper RH, Auwerx J, Franken P, Tafti M (2012) Key electrophysiological, molecular, and metabolic signatures of sleep and wakefulness revealed in primary cortical cultures. J Neurosci 32:12506-12517.

Hjelmstad GO, Nicoll RA, Malenka RC (1997) Synaptic refractory period provides a measure of probability of release in the hippocampus. Neuron 19:1309-1318.

Huang EP (1998) Synaptic plasticity: Going through phases with LTP. Curr Biol 8:R350-R352.

Jimbo Y, Robinson HP, Kawana A (1998) Strengthening of synchronized activity by tetanic stimulation in cortical cultures: Application of planar electrode arrays. IEEE Trans Biomed Eng 45:1297-1304.

Jimbo Y, Tateno T, Robinson HP (1999) Simultaneous induction of pathway-specific potentiation and depression in networks of cortical neurons. Biophys J 76:670-678.

Ju H, Dranias MR, Banumurthy G, VanDongen AM (2015) Spatiotemporal memory is an intrinsic property of networks of dissociated cortical neurons. J Neurosci 35:4040-4051.

Kerschensteiner D (2014) Spontaneous network activity and synaptic development. Neuroscientist 20:272-290.

Legenstein R, Maass W (2007) Edge of chaos and prediction of computational performance for neural circuit models. Neural Netw 20:323-334.

Lignani G, Ferrea E, Difato F, Amarù J, Ferroni E, Lugarà E, Espinoza S, Gainetdinov RR, Baldelli P, Benfenati F (2013) Long-term optical stimulation of channelrhodopsin-expressing neurons to study network plasticity. Front Mol Neurosci 6:22.
Litke AM, Bezayiff N, Chichilnisky EJ, Cunningham W, Dabrowski W, Grillo AA, Grivich M, Grybos P, Hottowy P, Kachiguine S, Kalmar RS, Mathieson K, Petrusca D, Rahman A, Sher A (2004) What does the eye tell the brain?: Development of a system for the large-scale recording of retinal output activity. IEEE Trans Nucl Sci 51:1434-1440.

Luhmann HJ, Sinning A, Yang JW, Reyes-Puerta V, Stüttgen MC, Kirischuk S, Kilb W (2016) Spontaneous neuronal activity in developing neocortical networks: From single cells to large-scale interactions. Front Neural Circuits 10:40.

Madhavan R, Chao ZC, Wagenaar DA, Bakkum DJ, Potter SM (2006) Multi-site stimulation quiets network-wide spontaneous bursts and enhances functional plasticity in cultured cortical networks. Conference proceedings of the Annual International Conference of the IEEE Engineering in Medicine and Biology Society 1:15931596.

Maeda E, Robinson H, Kawana A (1995) The mechanisms of generation and propagation of synchronized bursting in developing networks of cortical neurons. J Neurosci 15:6834-6845.

Maeda E, Kuroda Y, Robinson HP, Kawana A (1998) Modification of parallel activity elicited by propagating bursts in developing networks of rat cortical neurones. Eur J Neurosci 10:488-496.

Massobrio P, Tessadori J, Chiappalone M, Ghirardi M (2015) In vitro studies of neuronal networks and synaptic plasticity in invertebrates and in mammals using multielectrode arrays. Neural Plast 2015:196195.

McVea DA, Murphy TH, Mohajerani MH (2016) Large scale cortical functional networks associated with slow-wave and spindle-burstrelated spontaneous activity. Front Neural Circuits 10:103.

Mitra A, Snyder AZ, Hacker CD, Pahwa M, Tagliazucchi E, Laufs H, Leuthardt EC, Raichle ME (2016) Human cortical-hippocampal dialogue in wake and slow-wave sleep. Proc Natl Acad Sci USA 113:E6868-E6876.

Nickerson RS (1965) Short-term memory for complex meaningful visual configurations: A demonstration of capacity. Can J Psychol 19:155-160.

Oliveira AM, Hawk JD, Abel T, Havekes R (2010) Post-training reversible inactivation of the hippocampus enhances novel object recognition memory. Learn Mem 17:155-160.

Park S, Kramer EE, Mercaldo V, Rashid AJ, Insel N, Frankland PW, Josselyn SA (2016) Neuronal allocation to a hippocampal engram. Neuropsychopharmacology 41:2987-2993.

Pöschel B, Manahan-Vaughan D (2005) Group II mGluR-induced long term depression in the dentate gyrus in vivo is NMDA receptor-independent and does not require protein synthesis. Neuropharmacology 49:1-12.

Potter SM, DeMarse TB (2001) A new approach to neural cell culture for long-term studies. J Neurosci Methods 110:17-24.

Ruaro ME, Bonifazi P, Torre V (2005) Toward the neurocomputer: Image processing and pattern recognition with neuronal cultures. IEEE Trans Biomed Eng 52:371-383.

Sharma M, Sajikumar S (2014) Metaplasticity of synaptic tagging and capture: Memory beyond the circle. In: Synaptic tagging and capture: From synapses to behavior, pp 197-211. New York: Springer.

Shepard RN (1967) Recognition memory for words, sentences, and pictures. J Verbal Learning Verbal Behav 6:156-163.

Squire LR, Wixted JT, Clark RE (2007) Recognition memory and the medial temporal lobe: A new perspective. Nat Rev Neurosci 8:872-883.

Standing L (1973) Learning 10,000 pictures. Q J Exp Psychol 25:207-222.

Subramoney A, Scherr F, Maass W (2019) Reservoirs learn to learn. arXiv:1909.07486v2.

Tanaka Y, Isomura T, Shimba K, Kotani K, Jimbo Y (2017) Neurogenesis enhances response specificity to spatial pattern stimulation in hippocampal cultures. IEEE Trans Biomed Eng 64:2555-2561.

Tateno T, Jimbo Y (1999) Activity-dependent enhancement in the reliability of correlated spike timings in cultured cortical neurons. Biol Cybern 80:45-55. 
van Pelt J, Wolters PS, Corner MA, Rutten WL, Ramakers GJ (2004) Long-term characterization of firing dynamics of spontaneous bursts in cultured neural networks. IEEE Trans Biomed Eng 51:2051-2062.

Vlahou EL, Thurm F, Kolassa I-T, Schlee W (2014) Restign-state slow wave power, healthy aging and cognitive performance. Sci Rep 4:1-6.

Vogt S, Magnussen S (2007) Expertise in pictorial perception: Eyemovement patterns and visual memory in artists and laymen. Perception 36:91-100.

Wagenaar DA, Madhavan R, Pine J, Potter SM (2005) Controlling bursting in cortical cultures with closed-loop multi-electrode stimulation. J Neurosci 25:680-688.

Wagenaar DA, Pine J, Potter SM (2006a) An extremely rich repertoire of bursting patterns during the development of cortical cultures. BMC Neurosci 7:11.

Wagenaar DA, Pine J, Potter SM (2006b) Searching for plasticity in dissociated cortical cultures on multi-electrode arrays. J Negat Results Biomed 5:16.
Winters BD, Bussey TJ (2005) Transient inactivation of perirhinal cortex disrupts encoding, retrieval, and consolidation of object recognition memory. J Neurosci 25:52-61.

Wixted JT (2007) Dual-process theory and signal-detection theory of recognition memory. Psychol Rev 114:152-176.

Xu J, Antion MD, Nomura T, Kraniotis S, Zhu Y, Contractor A (2014) Hippocampal metaplasticity is required for the formation of temporal associative memories. J Neurosci 34:1676216773.

Yizhar O, Fenno LE, Davidson TJ, Mogri M, Deisseroth K (2011) Optogenetics in neural systems. Neuron 71:9-34.

Yonelinas AP (2002) The nature of recollection and familiarity: A review of 30 years of research. J Mem Lang 46:441-517.

Zhang X, Ju H, Penney TB, VanDongen AMJ (2017) Familiarity detection is an intrinsic property of cortical microcircuits with bidirectional synaptic plasticity. eNeuro 4. 